Glycoconjugates

\title{
Synthesis of Constrained C-Glycosyl Amino Acid Derivatives Involving 1,3-Dipolar Cycloaddition of Cyclic Nitrone as Key Step
}

\author{
Florian Rouzier, ${ }_{t}^{[a]}$ Rosanne Sillé, ${ }^{[a]}$ Ophélie Montiège ${ }^{[a]}$ Arnaud Tessier ${ }_{f}^{[b]}$ Muriel Pipelier ${ }^{[b]}$ \\ Gilles Dujardin, ${ }^{[a]}$ Arnaud Martel, ${ }^{[a]}$ Arnaud Nourry, ${ }^{*[a]}$ and Stéphane Guillarme*[a]
}

Abstract: An efficient two-step strategy for the synthesis of constrained C-glycosyl amino acid derivatives from C-vinylglycosides involving a 1,3-dipolar cycloaddition using $\mathrm{L}-(-)$ menthone-derived nitrone as the key step is described. After optimization of 1,3-dipolar cycloaddition conditions, various C-vinylglycosides were tested leading exclusively to one dia- stereoisomer of the corresponding cycloadduct in good to excellent yields. The total facial selectivity observed was also studied by DFT calculations. Original conformationally restricted C-glycosyl amino acid derivatives ( 8 examples) were isolated after simple cleavage of the chiral auxiliary.

\section{Introduction}

Glycoconjugates such as glycoproteins and glycolipids are fundamental for many important biological processes including cell-cell, bacterium-cell, and virus-cell recognition, inflammation, signal transduction, fertilization, immune defense and they are major components of the outer surface of mammalian cells." ${ }^{[1]}$ In glycoproteins, the carbohydrate part is generally covalently linked to the protein backbone through an oxygen atom (serine, threonine, tyrosine) or a nitrogen atom (asparagine). A glycosyl amino acid known as $T_{N}$ antigen ( $\alpha-D-G a I N A C$ linked to Ser/Thr) has particularly attracted a great deal of interest since this antigen has been identified as tumor-associated carbohydrate antigens (TACA) which are highly expressed on tumor cells. ${ }^{[2]}$ The incorporation of such a structure in an anticancer vaccine in order to stimulate the immune system to fight tumor cells became a therapeutic strategy. However, the main drawback of such carbohydrate-derived molecules is their low metabolic or chemical stability due to the hydrolytic lability of the glycosidic bond limiting their use as potential drug candidates.

It is now well established that the replacement of the anomeric oxygen (or nitrogen) atom of the glycosidic bond by a methylene group provide C-glycoside analogs as stable mim-

[a] Dr. F. Rouzier, R. Sille, O. Montiege, Dr. G. Dujardin, Prof. Dr. A. Martel, Dr. A. Nourry, Dr. S. Guillarme

Institur des Molécules et Matérioux du Mans, Le Mans Université and CNRS UMR 6283,

Avenue 0 . Messiaen, 72085 Le Mans cedex 9. France

E.mail: stephane.guillarme@univ-lemans.fr arnaud.nourry@univ-lemans.ir

(b) Dr. A. Tessier, Dr. M. Pipelier

CEISAM, Université de Nontes and CNRS UMR 6220.

2. rue de la Houssiniere, 44322 Nantes cedex 3. France ics. ${ }^{[3]}$ In that context, numerous research groups have focused their attention on the development of new methodologies to prepare C-glycosyl amino acids. These methods have already been reviewed ${ }^{[4]}$ and most of them were focused on C-glycosyl serine. The latter can be synthesized involving, as a key step, $\mathrm{Sml}_{2}$-promoted C-glycosylation, ${ }^{[5]}$ nucleophilic addition on carbohydrate derivative, ${ }^{[6]}$ metathesis reaction, ${ }^{[7]}$ Suzuki coupling $^{[8]}$ and Wittig-Horner ${ }^{[9]}$ or Ramberg-Bäcklund ${ }^{10]}$ olefination. Construction of the amino acid moiety on a C-glycoside derivative has also been performed. ${ }^{[11}$ In the case of $C$-glycosyl threonine, ${ }^{[8,12]}$ and C-glycosyl tyrosine, ${ }^{[8,13]}$ only a few syntheses have been described.

In addition, the recognition of glycoproteins by the biological target is related to the presentation mode of the glycan part. In order to modulate the glycan presentation, the syntheses of constrained glycosyl amino acids have been reported. For example, Halcomb et al. prepared a conformationally constrained $T_{N}$ antigen by incorporating the side chain into a small ring $^{[14]}$ or the $C \alpha$ of the amino acid in a six-membered ring. ${ }^{[15]}$ Dondoni et al. focused particularly on the synthesis of C-glycosyl amino acids with the amino acid side chains linked to the carbohydrate moiety via linkers such as triazole or isoxazole, ${ }^{[16]}$ pyridine, ${ }^{[17]}$ and tetrazole. ${ }^{[18]}$ Avenoza and Peregrina demonstrated that their conformationally restricted $T_{N}$ antigen analogs were recognized by the SBL lectin (GaINAC specific lectin).!19! In addition, Nativi and Renaudet reported the first anti-cancer vaccine containing structurally constrained $T_{N}$ antigen analog which presented a robust and longlasting $T_{N}$-specific antibodies response through a mechanism mediated by $B$ cells leading to improved protection against cancer in mice. ${ }^{[20]}$ Herein, we describe the synthesis of original constrained C.glycosyl amino acid derivatives in different series involving a 1,3-dipolar cycloaddition between a nitrone ${ }^{[21]}$ and C-vinylglycoside as key step (Scheme 1). 


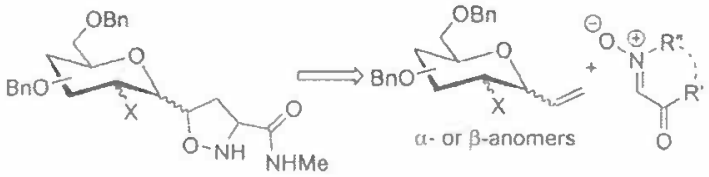

Gal, Glc, Man and GaINAc series

Scheme 1. General strategy for the synthesis of constrained C-glycosyl amino acid derivatives.

This kind of cycloaddition has already been performed between nitrones and carbohydrate-derived alkenes ${ }^{[22-24]}$ as dipolarophiles but, to the best of our knowledge, the use of carbohydrate-containing a vinyl group in pseudo-anomeric position has not been described ${ }^{[25}$

\section{Results and Discussion}

We recently reported an efficient synthesis of C-vinylglycoside in different series from the corresponding alkynes on gram scale. ${ }^{[26]}$ The reactivity of such dipolarophiles towards the ethyl glyoxylate-derived nitrone ${ }^{[27]} 2$ was first evaluated. The reaction between this nitrone and both $\alpha$ - and $\beta$-anomers of $C$-vinylgalactoside $1 \mathrm{a}$ and $1 \mathrm{~b}$ in toluene at $100^{\circ} \mathrm{C}$ led to the expected adducts $3 a$ and $3 b$ as a mixture of isomers. Separation of isomers by chromatography on silica, although tough, led in both cases to two isolated diastereoisomers identified as a trans and a cis one, in a $3: 1$ ratio for the cycloadduct $3 a$ ( $76 \%$ global yield) and in a 2:1 ratio for the cycloadduct $3 \mathrm{~b}$ ( $64 \%$ global yield) (Scheme 2). This predominance of the trans adduct was confirmed by NOESY analyses of each isolated diastereoisomer in the two series (see Supporting Information). While trans diastereoisomers are undoubtedly formed via an exo approach (the less congested) to the $E$ nitrone (the more reactive), ${ }^{[21]}$ the cis diastereoisomers can result from either an exo approach to the $Z$ nitrone or an endo approach to the $E$ nitrone (or from both) In order to control the cis/trans selectivity, 1,3-dipolar cycloaddition was performed with the configurationally stable cyclic nitrone $4^{[28]}$ The latter was prepared in three steps from methyl glycinate in $70 \%$ overall yield (See supporting information).
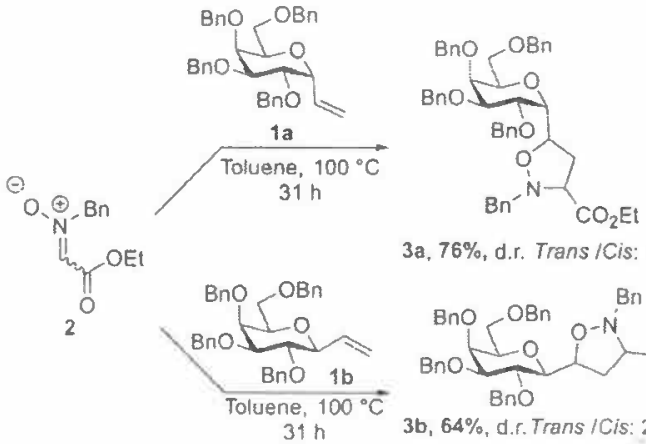

3a, $76 \%$, d.r. Trans / Cis: $3: 1$

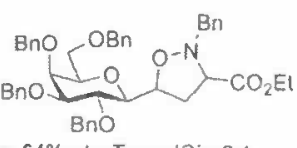

$3 \mathrm{~b}, 64 \%$, d.f. Trans /Cis: $2: 1$

Scheme 2. Reaction between nitrone $\mathbf{2}$ and $C$-vinylgalactoside $1 \mathrm{a}$ and $1 \mathrm{~b}$.

Heating of the cyclic nitrone with $\beta$-C-vinylgalactoside $1 \mathrm{~b}$ in toluene at $110^{\circ} \mathrm{C}$ for 70 hours led to two cycloadducts 5 in $54 \%$ yield in a 2:1 ratio (Scheme 3 ). The two compounds were clearly identified as the two trans diastereoisomers from the NOESY spectra. The two trans isomers were derived from an exo approach but no facial discrimination was observed, showing that the chirality of the dipolarophile does not bring any selectivity.

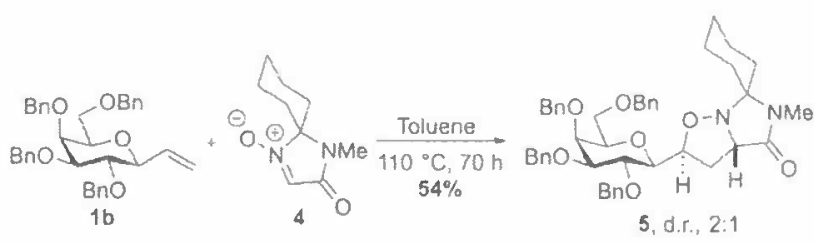

Scheme 3. Reaction between nitrone 4 and C-vinylgalactoside ib

To improve the facial selectivity, we focused our attention on chiral cyclic nitrones bearing a bulky group in order to mask one face of the dipole. The reactivity of the Katagiri's nitrone $6 a^{[29]}$ with the $\beta$-C-vinylgalactoside $1 \mathrm{~b}$ was first evaluated. Whatever the activation mode (microwave or Lewis acid), the cycloaddition did not occur (Table 1, entries 1-2). Having in mind the reactivity of the chiral menthone-derived nitrone $6 \mathrm{~b}$ with C-, O-, and S-allyl glycosides reported by either Altenbach et al. ${ }^{[23]}$ or Praly et al., ${ }^{[30]}$ we then performed the 1,3-dipolar cycloaddition with this dipole. The nitrone $6 \mathrm{~b}$ was prepared in two steps from L-menthone in a $27 \%$ overall yield. As a first attempt, the procedure described by Altenbach et al. ${ }^{[23]}$ i.e. heating of one equivalent of the nitrone $6 \mathrm{~b}$ with one equivalent of $\beta$-C-vinylgalactoside $\mathbf{1 b}$ in toluene at reflux for 72 hours was reproduced, leading to the formation of only one diastereoisomer of cycloadduct $7 b$ in $36 \%$ yield (entry 3 ). In our case, the reaction was incomplete after 72 hours. This can be explained by the use of a more hindered olefin compared with those involved by Altenbach. The NOESY analysis of the cycloadduct allowed clearly the identification of a trans diastereoisomer as a result of an exo approach.

Table 1. Optimization of the 1,3-dipolar cycloaddition of nitrone 6 with $\beta \cdot C$ vinylgalactoside $1 \mathrm{~b}$

$\begin{array}{llll}6 & \\ 6 \mathrm{~b}, \mathrm{X}=\mathrm{NMe} \\ 1 \text { eq. }\end{array}$

Unfortunately, all attempts to obtain a crystailine structure of the unprotected cycloadduct, ${ }^{[31]}$ in order to determine absolute configurations of the two new stereogenic centers, failed. 
When the reaction was performed in p-xylene at $140{ }^{\circ} \mathrm{C}$ for 30 hours, increasing amounts of degradation products were observed and the same diastereoisomer was isolated in lower $27 \%$ yield (entry 4 ). Reaction times could be reduced by heating the reaction mixture in a sealed tube at $140^{\circ} \mathrm{C}$ or $160^{\circ} \mathrm{C}$ and the yield was significantly improved to $66 \%$ (entries 5-6). The same range of yield was observed when the reaction was performed at $140^{\circ} \mathrm{C}$ under microwave activation but the reaction time was halved (entry 8). Whatever the activation mode, heating at a higher temperature has resulted in a drop in the yields (entries 7 and 9). Increase of the concentration of the reaction mixture (from $0.1 \mathrm{M}$ to $0.5 \mathrm{M}$ ) allowed to isolate the cycloadduct in $75 \%$ yield with only 2 hours of reaction time (entry 10 ). It is noteworthy that traces of nitrone $6 \mathrm{~b}$ were recovered along with the cycloadduct after purification since the two compounds had the same $R_{\text {f. }}$. Finally, the use of only 0.9 equivalent of nitrone $6 \mathrm{~b}$ under the optimal conditions (described in entry 10) furnished a pure cycloadduct without any trace of nitrone $6 \mathrm{~b}$ in $72 \%$ yield.

The optimal conditions were then applied to different $C$-vinylglycosides. When the reaction was implemented for the $\beta$-anomers in glucose and mannose series, the corresponding cycloadducts 8 and 9 were isolated, after 2 hours of reaction, in excellent yields of 91 and $90 \%$ respectively (Scheme 4). In the case of $\alpha$-anomers in galactose, glucose, and mannose series, a longer reaction time (6 hours) was required to complete the reaction and to access the corresponding cycloadducts 10-12 with good yields (Scheme 4). Both anomers of C-vinylgalactosamines were also subjected to the cycloaddition with nitrone $6 \mathrm{~b}$ leading to the cycloadduct 13 and 14 after 8 hours of reaction in 77 and $63 \%$ yields respectively (Scheme 4). NOESY analyses of the cycloadducts showed that only one trans diastereoisomer was formed.
Among all cycloadducts, only cycloadduct 9 showed, on the 'H NMR spectrum, three doublet signals at $0.78,0.85$, and $0.91 \mathrm{ppm}$ as expected for the three corresponding $\mathrm{CH}_{3}$ of the menthyl moiety. COSY NMR experiment allowed us to identify the two $\mathrm{CH}_{3}$ of the isopropyl group which were located at 0.78 and $0.85 \mathrm{ppm}$ (Figure 1). Furthermore, NOESY NMR spectrum highlighted a cross-peak between one $\mathrm{CH}_{3}$ of the isopropyl group and $\mathrm{H}-3^{\prime}$, thus indicating an $\mathrm{S}$ absolute configuration for the $C-3^{\prime}$ stereogenic center (Figure 1). As already mentioned, due to a trans relationship between $\mathrm{H}-\mathrm{l}^{\prime}$ and $\mathrm{H}-3^{\prime}, \mathrm{S}$ absolute configuration could be assigned for the $\mathrm{C}-\gamma^{\prime}$ stereogenic center. This diastereoisomer could solely be obtained by an exo approach via the si face of the nitrone. This total facial selectivity could be due to the strong discrimination imposed by the isopropyl group of the chiral auxiliary of the nitrone $6 \mathrm{~b}$. The same stereoselectivity outcome was obtained compared to the one observed by Westerman ${ }^{[23]}$ and Praly ${ }^{[30]}$ groups with other dipolarophiles suggesting that the stereoselectivity is exclusively dependent on the stereochemistry of this nitrone.

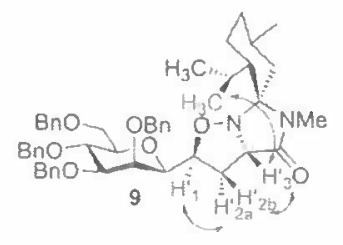

Figure 1. Main NOESY NMR cross-peaks of cycloadduct 9

In order to study the facial selectivity, DFT calculations were performed using Gaussian $09^{[32]}$ at M06-2X/6-311+G(d,p) level. ${ }^{[33]}$ The influence of the solvent (toluene) was studied using the SMD model. ${ }^{[34]}$ With the aim of limiting the possible conformations and the calculation time, the study and the cal-
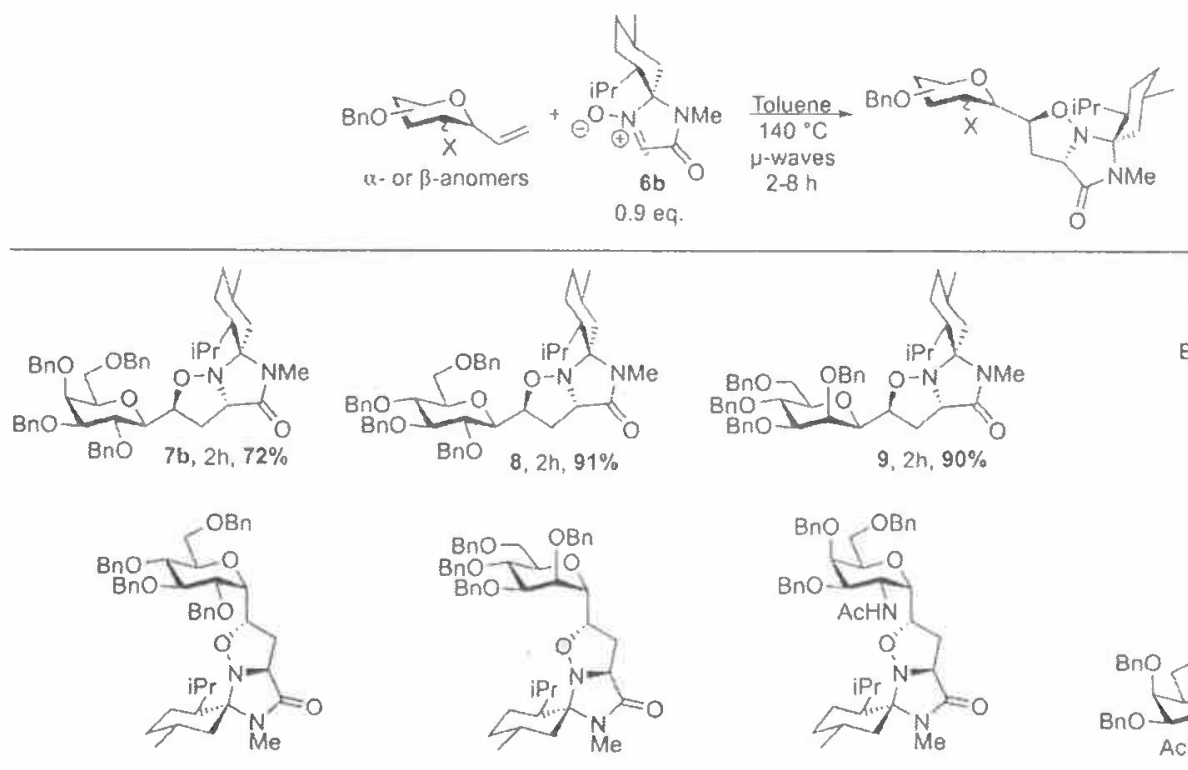

$13,8 \mathrm{~h}, 77 \%$

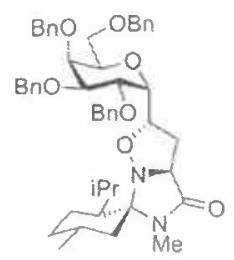

$10,6 \mathrm{~h} .73 \%$

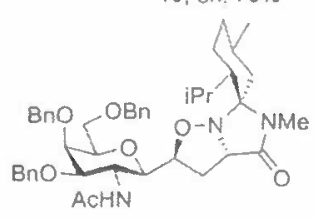

14,8 h, $63 \%$ 
culations were carried out with the a-C-vinylgalactoside for which the benzyl groups were replaced by methyl groups. ${ }^{[35]}$ At the top of Scheme 5 are depicted the four cycloadducts that could be produced by the reaction of $1 a^{\prime}$ and $6 b$ according to exo/endo approaches and Si/Re face of the nitrone. The energy profile calculated for the four diastereoisomers (Scheme 5) describes the Gibbs free energy at $413 \mathrm{~K}$ (reaction temperature, $140^{\circ} \mathrm{C}$ ). Five transition states were found during the study and the most favorable approach was the exo one from the si face of the nitrone (the less hindered). Indeed, the two lowest transition states observed for this approach (see Scheme 5) corresponded to a different orientation of the vinytic bound which benefits from a larger rotation degree than in the other three approaches. It is noteworthy that the contribution of the hindered face of the nitrone can be considered as the major factor favoring the two approaches from the Si face of this nitrone.
This total facial selectivity is therefore essentially due to the strong discrimination imposed by the isopropyl group of the chiral auxiliary of the nitrone $6 \mathrm{~b}$. These theoretical results are therefore in complete agreement with the experimental ones. Thus, taking into account that the stereoselectivity is only driven by the nitrone, we assumed that all the cycloadducts (from all series) have been formed with the same stereoselectivity.

Finally, in order to complete our study, the cleavage of the chiral auxiliary was implemented on all cycloadducts. Treatment of cycloadduct $\mathbf{7 b}$ with an aqueous hydrochloric acid solution in presence of acetic acid at $80^{\circ} \mathrm{C}$ provided the constrained C- galactosyl amino acid derivative 15 in $67 \%$ yield. The same procedure was applied to all the cycloadducts leading to the corresponding original constrained C-glycosyl amino acid derivatives 16-22 in moderate to good yields (Scheme 6).
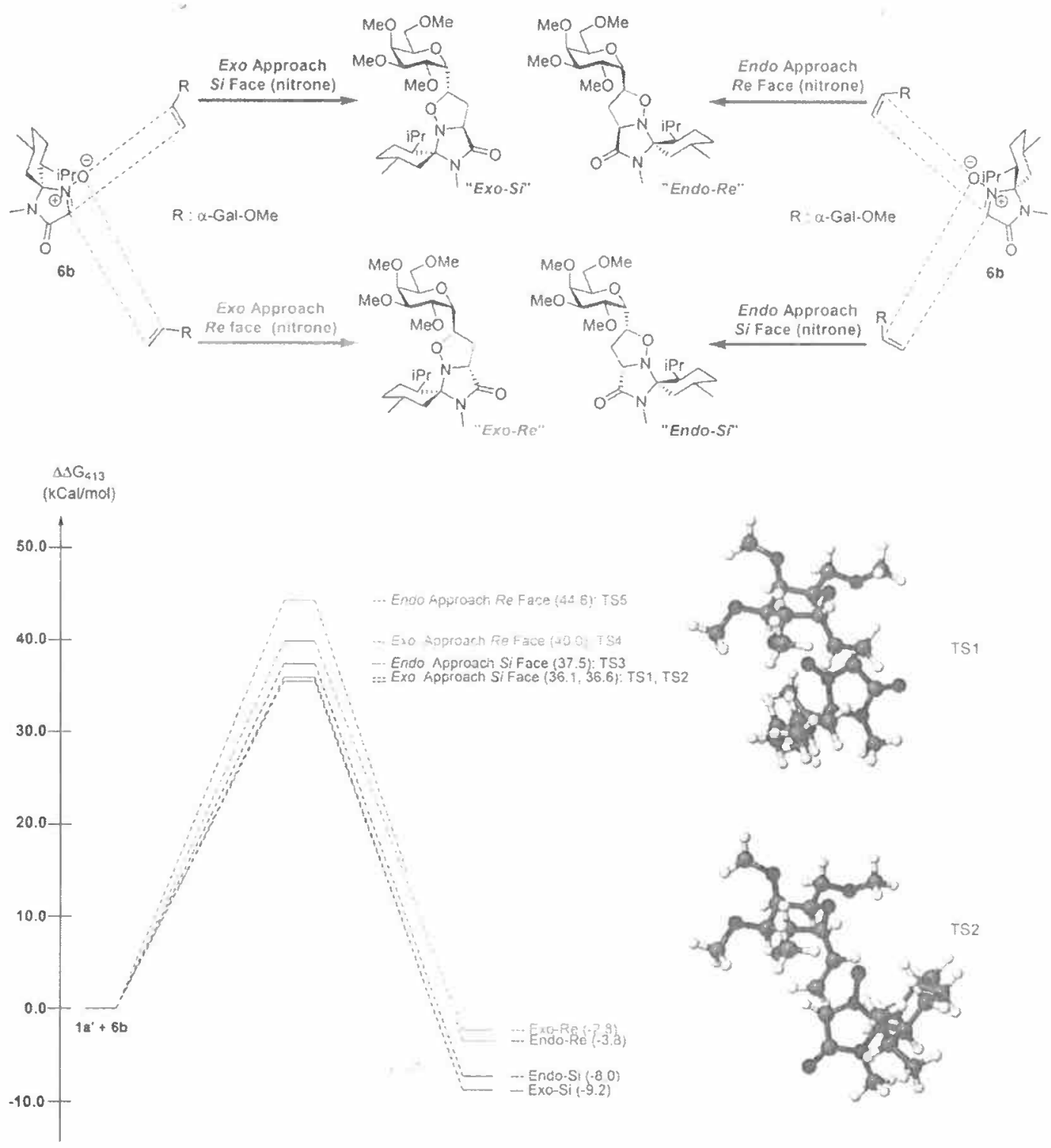

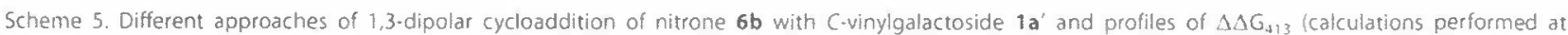
M06-2X/6-311+G(d,p) SMD (toluene) level). 

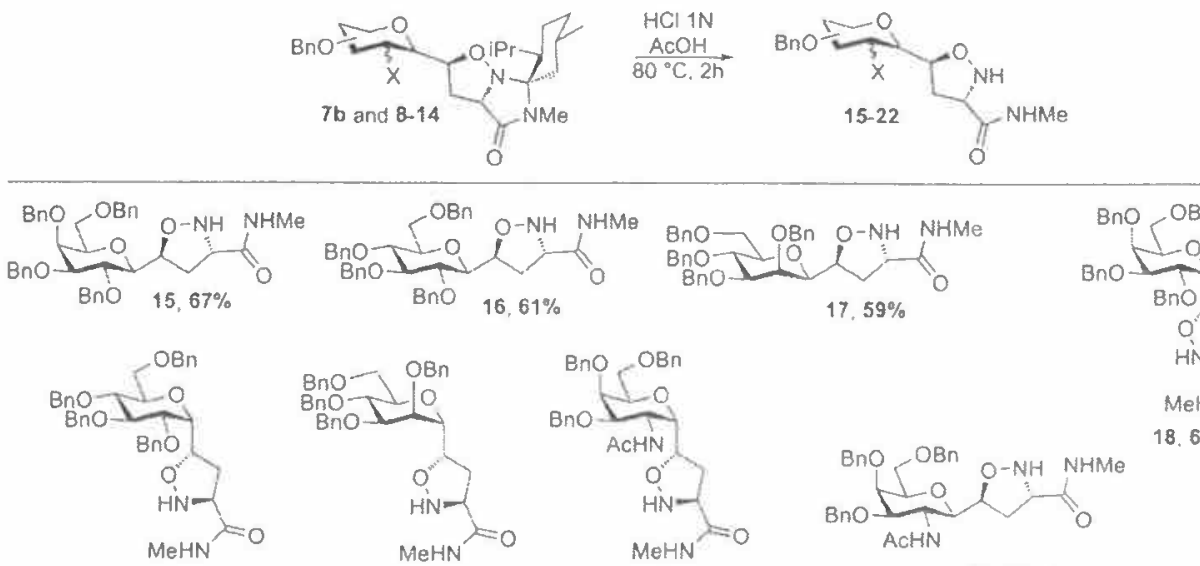

$19,73 \%$

$20.62 \%$

$21,58 \%$

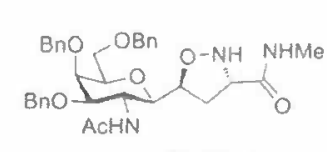

$22.55 \%$

Scheme 6 . Access to constrained $C$-glycosyl amino acid derivatives.

\section{Conclusion}

In summary, we described a two-step synthesis of original conformationally restricted C-glycosyl amino acid derivatives in different series involving 1,3-dipolar cycloaddition between L-(-)menthone-derived nitrone and (-vinylglycosides as key step. The 1,3-dipolar cycloaddition reaction was totally regio- and stereoselective leading to only one diastereoisomer in good to excellent yields whatever the dipolarophile. Based on both NMR experiments and DFT calculations, we showed that all the cycloadducts could solely be obtained by an exo approach via the $5 i$ face of the nitrone with total facial discrimination. The cleavage of the chiral auxiliary allowed to access new constrained C-glycosyl amino acid- derivatives as interesting building blocks. Opening of the isoxazolidine ring and functionalization of the pseudo-anomeric position, offering the possibility to prepare different analogs of C-glycosyl serine, are currently ongoing

\section{Experimental Section}

All experiments were carried out under argon with anhydrous solvents in dried glassware. THF was dried with activated alumina on a dry station purchased from Innovative Technologies. Commercially available materials were used without further purification. Flash chromatography was performed on silica gel (40-63 $\mu \mathrm{m}$ from Macherey-Nagel) using Reveleris $X^{2}$ Grace apparatus. Analytical TLCs were carried out on pre-coated silica gel 60 F254 from MachereyNagel. Optical rotations were measured using a Jasco P2000 at the sodium $D$ line $(=589 \mathrm{~nm})$ with a $1-\mathrm{dm}$ path length cell at $25^{\circ} \mathrm{C}$. Melting points were measured using Büchi B-545 apparatus. NMR spectra were recorded with a Bruker Avance 400. Chemical shifts are reported in ppm from TMS as the internal standard for the ' $\mathrm{H}$ NMR spectrum and from the residual peaks of the solvent $\left(\mathrm{CDCl}_{3}\right)$ for ${ }^{13} \mathrm{C}$ NMR spectrum. Structural assignments of the isolated compounds were based on ${ }^{1} \mathrm{H},{ }^{13} \mathrm{C}, \mathrm{COSY}$, and HSQC NMR experiments (see Supporting Information for numbering of assignment). Highresolution mass spectroscopy was performed with a Bruker microTOF QIII mass spectrometer using ESI techniques.

General Procedure for $[3+2]$ Cycloaddition with Nitrone 6b: C-vinylglycoside ( 1 equiv.) and nitrone $(0.9$ equiv.) were dissolved in toluene $(3 \mathrm{~mL}, \mathrm{C}=0.5 \mathrm{~m})$. The reaction mixture was stirred under microwaves at $140^{\circ} \mathrm{C}$ for $2-8 \mathrm{~h}$ then concentrated under reduced pressure.

Cycloadduct (7b): Following the general procedure (2 h), $\beta$-C-vinylgalactoside (304 $\mathrm{mg}, 0.553 \mathrm{mmol}$ ) afforded cycloadduct $7 \mathrm{~b}$ ( $273 \mathrm{mg}, 0.35 \mathrm{mmol}, 72 \%$ ) after purification by column chromatography (cyclohexane/EtOAC, 95:5 to 87:13). Colorless oil. $R_{\mathrm{f}}=0.27$ (cyclohexane/EtOAC, 8:2). $[\alpha]_{D}^{25}+28.8$ ( $\mathrm{C}$ 1.02, $\left.\mathrm{CHCl}_{3}\right)$. HRMS (ESI $)^{+}$). Calculated for $\mathrm{C}_{49} \mathrm{H}_{61} \mathrm{~N}_{2} \mathrm{O}_{7}[\mathrm{M}+\mathrm{H}]^{+}: m / z=789.4473$, found 789.4442. ' $\mathrm{H}$ NMR ( $400 \mathrm{MHz}, \mathrm{CDCl}_{3}$ ): $\delta 7.37-7.21(\mathrm{~m}, 20 \mathrm{H}, \mathrm{ArH}), 4.95$ and 4.58 ( $\mathrm{AB}$ syst, $)=11.3 \mathrm{~Hz}, 2 \mathrm{H}, \mathrm{CH}_{2} \mathrm{Ph}$ ), 4.92 and 4.53 ( $\mathrm{AB}$ syst, $J=10.8 \mathrm{~Hz}, 2 \mathrm{H},\left(\mathrm{H}_{2} \mathrm{Ph}\right.$ ), 4.75 and 4.65 ( $\mathrm{AB}$ syst, $J=11.7 \mathrm{~Hz}, 2 \mathrm{H}$, $\mathrm{CH}_{2} \mathrm{Ph}$ ), 4.47-4.40 ( $\left.\mathrm{m}, 2 \mathrm{H}, \mathrm{CH}_{2} \mathrm{Ph}\right), 4.08-4.03\left(\mathrm{~m}, 1 \mathrm{H}, \mathrm{H}-1^{\prime}\right), 4.01$ (d, $J=2.5 \mathrm{~Hz}, 1 \mathrm{H}, \mathrm{H}-4), 3.92\left(\mathrm{~d}, J=8.7 \mathrm{~Hz}, 1 \mathrm{H}, \mathrm{H} \cdot 3^{\prime}\right), 3.69(\mathrm{~d}, J=9.3 \mathrm{~Hz}$, $1 \mathrm{H}, \mathrm{H}-2), 3.63-3.50(\mathrm{~m}, 5 \mathrm{H}, \mathrm{H}-1, \mathrm{H}-3, \mathrm{H}-5, \mathrm{H}-6), 2.70\left(\mathrm{~s}, 3 \mathrm{H}, \mathrm{NCH}_{3}\right.$ ), 2.69-2.62 (m, 1H, H-2'), 2.45-2.39 ( $\left.m, 1 H, H-2^{\prime}\right), 2.08-1.99(m, 1 H)$, $1.94-1.88(\mathrm{~m}, 1 \mathrm{H}), 1.79-1.65(\mathrm{~m}, 2 \mathrm{H}), 1.58-1.50(\mathrm{~m}, 1 \mathrm{H}), 1.48-1.34$ $(\mathrm{m}, 1 \mathrm{H}), 1.32-1.24(\mathrm{~m}, 1 \mathrm{H}), 1.17(\mathrm{t})=,12.4 \mathrm{~Hz}, 1 \mathrm{H}), 0.86-0.78(\mathrm{~m}$, 10H). ${ }^{13} \mathrm{C} \mathrm{NMR}\left(100.6 \mathrm{MHz} \mathrm{CDCl}_{3}\right): \delta=173.3(\mathrm{C}=0), 139.1(\mathrm{C}), 138.3$ (C), $138.2(\mathrm{C}), 138.0(\mathrm{C}), 128.5(3 \mathrm{CH}), 128.2(2 \mathrm{CH}), 128.0(\mathrm{CH}), 127.8$ $(2 \mathrm{CH}), 127.7(\mathrm{CH}), 127.6(\mathrm{CH}), 127.4(2 \mathrm{CH}), 89.7(\mathrm{C}), 84.9(\mathrm{C} 3), 76.9$ (C1 or $\mathrm{Cl}^{\prime}$ ), 76.9 (C1 or $\mathrm{C} 1$ ), 76.5 (C2 or $\mathrm{C} 5$ ), 76.4 (C2 or $\mathrm{C5}$ ), 75.5 $\left(\mathrm{CH}_{2}\right), 74.4\left(\mathrm{CH}_{2}\right), 73.5\left(\mathrm{CH}_{2}\right), 73.4(\mathrm{C} 4), 72.1\left(\mathrm{CH}_{2}\right), 68.2(\mathrm{C} 6), 66.2$ $\left(\mathrm{C}^{\prime}\right), 48.3(\mathrm{CH}), 40.5\left(\mathrm{CH}_{2}\right), 34.8\left(\mathrm{CH}_{2}\right), 32.6\left(\mathrm{C}^{\prime}\right), 29.4(\mathrm{CH}), 26.0$ $\left(\mathrm{CH}_{3}-\mathrm{N}\right), 24.4(\mathrm{CH}), 24.2\left(\mathrm{CH}_{3}\right), 22.4\left(\mathrm{CH}_{3}\right), 22.2\left(\mathrm{CH}_{2}\right), 18.4\left(\mathrm{CH}_{3}\right)$.

Cycloadduct ( 8 ): Following the general procedure (2 h), $\beta-C$-vinylglucoside $(373 \mathrm{mg}, 0.68 \mathrm{mmol}$ ) afforded cycloadduct 8 (440 $\mathrm{mg}$, $0.55 \mathrm{mmol}, 91 \%$ ) after purification by column chromatography (cyclohexane/EtOAC, 95:5 to 87:13). Colorless oil. $R_{i}=0.28$ (cyclohexane/EtOAC, 8:2). [ ( $\mathrm{A}]_{0}^{25}+31.1\left(\mathrm{C} \mathrm{0.99}, \mathrm{CHCl}_{3}\right)$. HRMS (ESI $)$ : Calculated for $\mathrm{C}_{49} \mathrm{H}_{61} \mathrm{~N}_{2} \mathrm{O}_{7}\left[\mathrm{M}+\mathrm{H}^{+}: m / z=789.4473\right.$, found 789.4463. ${ }^{\mathrm{H}} \mathrm{H} \mathrm{NMR}$ $\left(400 \mathrm{MHz}_{1} \mathrm{COCl}_{3}\right): \partial 7.34-7.17(\mathrm{~m}, 2 \mathrm{OH}, \mathrm{ArH}), 4.92$ and 4.87 (AB syst, $\left.J=10.9 \mathrm{~Hz}, 2 \mathrm{H}, \mathrm{CH}_{2} \mathrm{Ph}\right), 4.87$ and 4.56 ( $\mathrm{AB}$ syst, $J=10.9 \mathrm{~Hz}, 2 \mathrm{H}$, $\left(\mathrm{H}_{2} \mathrm{Ph}\right.$ ), 4.80 and 4.61 ( $\mathrm{AB}$ syst, $J=10.8 \mathrm{~Hz}, 2 \mathrm{H}, \mathrm{CH}_{2} \mathrm{Ph}$ ), 4.60 and 4.52 ( $\mathrm{AB}$ syst, $\left.)=12.0 \mathrm{~Hz}, 2 \mathrm{H}, \mathrm{CH}_{2} \mathrm{Ph}\right), 4.08-4.03\left(\mathrm{~m}, \mathrm{H}, \mathrm{H}-1^{\prime}\right), 3.94$ $\left(d, J=8.5 \mathrm{~Hz}, 1 \mathrm{H}, \mathrm{H}-3^{\prime}\right), 3.76-3.63(\mathrm{~m}, 4 \mathrm{H}, \mathrm{H}-3, \mathrm{H}-4, \mathrm{H}-6), 3.57-3.52$ (m, $1 \mathrm{H}, \mathrm{H}-1), 3.44-3.39(\mathrm{~m}, 1 \mathrm{H}, \mathrm{H}-5), 3.39-3.33(\mathrm{~m}, 1 \mathrm{H}, \mathrm{H}-2), 2.71$ (s, $\left.3 \mathrm{H}, \mathrm{N} \mathrm{CH} \mathrm{CH}_{3}\right), 2.71-2.63\left(\mathrm{~m}, \mathrm{iH}, \mathrm{H}-\mathrm{2}^{\prime}\right), 2.48-2.42\left(\mathrm{~m}, 1 \mathrm{H}, \mathrm{H}-2^{\prime}\right), 2.09$ $1.99(\mathrm{~m}, 1 \mathrm{H}), 1.97-1.91(\mathrm{~m}, 1 \mathrm{H}), 1.82-1.67(\mathrm{~m}, 2 \mathrm{H}), 1.60-1.53(\mathrm{~m}, 1 \mathrm{H})$, $1.44-1.35(\mathrm{~m}, 1 \mathrm{H}), 1.24-1.29(\mathrm{~m}, 1 \mathrm{H}), 1.18(\mathrm{t}, J=12.7 \mathrm{~Hz}, 1 \mathrm{H}), 0.93-$ $0.79(\mathrm{~m}, 10 \mathrm{H}) .{ }^{13} \mathrm{C} \mathrm{NMR}\left(100.6 \mathrm{MHz} \mathrm{CDCl}_{3}\right): \delta=173.3(\mathrm{C}=0), 138.4$ (C), $138.3(\mathrm{C}), 138.1(\mathrm{C}), 137.7(\mathrm{C}), 128.4(2 \mathrm{CH}), 128.3(2 \mathrm{CH}), 127.9$ $(3 \mathrm{CH}), 127.7(\mathrm{CH}), 127.6(3 \mathrm{CH}), 127.5(\mathrm{CH}), 89.7(\mathrm{C}), 87.1(\mathrm{C} 3$ or $\mathrm{C} 4)$, $79.6(\mathrm{C} 2), 78.8(\mathrm{C} 5), 78.1$ ( $\mathrm{C} 3$ or $(4), 76.7$ ( $\mathrm{C}$ ) or $\mathrm{Cl}$ ), $76.7\left(\mathrm{Cl}\right.$ or $\left.\mathrm{C} 1^{\prime}\right)$, 
$75.5\left(\mathrm{CH}_{2}\right), 75.2\left(\mathrm{CH}_{2}\right), 74.9\left(\mathrm{CH}_{2}\right), 73.4\left(\mathrm{CH}_{2}\right), 68.8(\mathrm{C} 6), 66.0\left(\mathrm{Cl}^{\prime}\right)$, $48.2(\mathrm{CH}), 40.4\left(\mathrm{CH}_{2}\right), 34.7\left(\mathrm{CH}_{2}\right), 32.8\left(\mathrm{Cl}^{\prime}\right), 29.4(\mathrm{CH}), 25.9\left(\mathrm{CH}_{3}-\mathrm{N}\right)$, $24.4(\mathrm{CH}), 24.3\left(\mathrm{CH}_{3}\right), 22.3\left(\mathrm{CH}_{3}\right), 22.3\left(\mathrm{CH}_{2}\right), 18.4\left(\mathrm{CH}_{3}\right)$.

Cycloadduct (9): Following the general procedure (2 h), $\beta$-C-vinylmannoside (230 mg, $0.42 \mathrm{mmol}$ ) afforded cycloadduct 9 (253 mg, $0.32 \mathrm{mmol}, 90 \%$ ) after purification by column chromatography (cyclohexane/EtOAC, 95:5 to 87:13). Colorless oil. $R_{\mathrm{f}}=0.27$ (cyclohexane/EtOAC, 8:2). $[a]_{0}^{25}+19.5$ ( 1 1.07, $\left(\mathrm{HCl}_{3}\right)$. HRMS $\left[\mathrm{ESI}{ }^{*}\right)$ : Calculated for $\mathrm{C}_{49} \mathrm{H}_{6}, \mathrm{~N}_{2} \mathrm{O},[\mathrm{M}+\mathrm{H}]^{+}: \mathrm{m} / \mathrm{z}=789.4473$, found 789.4473. ${ }^{1} \mathrm{H}$ NMP (400 $\mathrm{MHz}_{2} \mathrm{CDCl}_{3}$ ): $\delta 7.37-7.18(\mathrm{~m}, 20 \mathrm{H}, \mathrm{ArH}), 5.04$ and 4.58 (AB syst, $J=11.4 \mathrm{~Hz}, 2 \mathrm{H},\left(\mathrm{H}_{2} \mathrm{Ph}\right.$ ), 4.86 and 4.56 (AB syst, $J=10.8 \mathrm{~Hz}_{1} 2 \mathrm{H}$, $\mathrm{CH}_{2} \mathrm{Ph}$ ), 4.75 (s, $2 \mathrm{H}, \mathrm{CH}_{2} \mathrm{Ph}$ ), 4.61 and 4.52 (AB syst, $J=12.0 \mathrm{~Hz}, 2 \mathrm{H}$ $\left(\mathrm{H}_{2} \mathrm{Ph}\right), 4.23-4.16\left(\mathrm{~m}, 1 \mathrm{H}, \mathrm{H}-1^{\prime}\right), 4.00-3.97(\mathrm{~m}, 1 \mathrm{H}, \mathrm{H}-2), 3.94-3.88$ $\left(\mathrm{m}, 2 \mathrm{H}, \mathrm{H}-3^{\prime}, \mathrm{H}-4\right), 3.77-3.65(\mathrm{~m}, 2 \mathrm{H}, \mathrm{H}-6), 3.60$ (dd, $J=9.4,2.7 \mathrm{~Hz}$, $1 \mathrm{H}, \mathrm{H}-3), 3.46-3.40(\mathrm{~m}, 1 \mathrm{H}, \mathrm{H}-5), 3.26(\mathrm{dd}, J=8.6,0.6 \mathrm{~Hz}, 1 \mathrm{H}, \mathrm{H}-1)$, 2.83-2.76 (m, $\left.1 \mathrm{H}, \mathrm{H}-2^{\prime}\right), 2.69\left(\mathrm{~s}, 3 \mathrm{H}, \mathrm{N}-\mathrm{CH}_{3}\right), 2.52-2.44\left(\mathrm{~m}, 1 \mathrm{H}, \mathrm{H}-2^{\prime}\right)$, $2.09-2.03(\mathrm{~m}, 1 \mathrm{H}), 2.00-1.90(\mathrm{~m}, 1 \mathrm{H}), 1.88-1.80(\mathrm{~m}, 1 \mathrm{H}), 1.68-1.59$ $(\mathrm{m}, 2 \mathrm{H}), 1.48-1.40(\mathrm{~m}, 1 \mathrm{H}), 1.38-1.33(\mathrm{~m}, 1 \mathrm{H}), 1.18(\mathrm{t}, J=12.3 \mathrm{~Hz}$, $1 \mathrm{H}), 0.95-0.91(\mathrm{~m}, 1 \mathrm{H}), 0.91\left(\mathrm{~d}, J=6.6 \mathrm{~Hz}, 3 \mathrm{H}, \mathrm{CH}_{3}\right), 0.85(\mathrm{~d}, J=$ $\left.6.6 \mathrm{~Hz}, 3 \mathrm{H}, \mathrm{CH}_{3}\right), 0.78\left(\mathrm{~d}, J=6.6 \mathrm{~Hz}, 3 \mathrm{H}, \mathrm{CH}_{3}\right) .{ }^{13} \mathrm{C} \mathrm{NMR}(100.6 \mathrm{MHz}$, $\left.\mathrm{CDCl}_{3}\right): \delta=173.2(\mathrm{C}=0), 139.2(\mathrm{C}), 138.6(2 \mathrm{C}), 138.5(\mathrm{C}), 128.6(\mathrm{CH})$, $128.4(2 \mathrm{CH}), 128.3(\mathrm{CH}), 128.2(\mathrm{CH}), 127.9(\mathrm{CH}), 127.8(2 \mathrm{CH}), 127.7$ $(\mathrm{CH}), 127.6(\mathrm{CH}), 127.4(2 \mathrm{CH}), 88.7(\mathrm{C}), 84.5(\mathrm{C} 3), 80.4(\mathrm{C} 5), 79.2(\mathrm{C})$, $75.5(\mathrm{C} 4), 75.3\left(\mathrm{CH}_{2}\right), 75.3(\mathrm{C} 2), 75.0\left(\mathrm{CH}_{2}\right), 73.7\left(\mathrm{CH}_{2}\right), 73.5\left(\mathrm{Cl}^{\prime}\right), 72.5$ $\left(\mathrm{CH}_{2}\right), 69.8(\mathrm{C} 6), 65.6\left(\mathrm{C}^{\prime}\right), 48.2(\mathrm{CH}), 40.9\left(\mathrm{CH}_{2}\right), 36.1\left(\mathrm{Cl}^{\prime}\right), 34.9\left(\mathrm{CH}_{2}\right)$, $29.8(\mathrm{CH}), 26.0\left(\mathrm{CH}_{3}-\mathrm{N}\right), 24.5(\mathrm{CH}), 24.3\left(\mathrm{CH}_{3}\right), 22.6\left(\mathrm{CH}_{2}\right), 22.4\left(\mathrm{CH}_{3}\right)$, $18.6\left(\mathrm{CH}_{3}\right)$

Cycloadduct (10): Following the general procedure (4 h), $\alpha \cdot C$-vinylgalactoside $(826 \mathrm{mg}, 1.50 \mathrm{mmol})$ afforded cycloadduct 10 (790 mg, $1.00 \mathrm{mmol}, 73 \%$ ) after purification by column chromatography (cyclohexane/EtOAc, $95: 5$ to 87:13). Colorless oil. $R_{f}=0.44$ (cyclohexane/EtOAC, 6:4). [a] $]_{D}^{25}+42.3\left(\mathrm{C} 1.0, \mathrm{CHCl}_{3}\right)$. HRMS $\left.(E S)^{+}\right)$: Calculated for $\mathrm{C}_{49} \mathrm{H}_{61} \mathrm{~N}_{2} \mathrm{O}_{7}\left[\mathrm{M}+\mathrm{H}^{+*}: \mathrm{m} / z=789.4473\right.$, found 789.4473. ' $\mathrm{H}$ NMR (400 MHz, $\left.\mathrm{COCl}_{3}\right): \delta 7.35-7.20(\mathrm{~m}, 20 \mathrm{H}, \mathrm{ArH}), 4.80$ and 4.56 (AB syst, $J=11.7 \mathrm{~Hz}, 2 \mathrm{H}, \mathrm{CH}_{2} \mathrm{Ph}$ ), 4.72 and 4.65 ( $\mathrm{AB}$ syst, $J=11.5 \mathrm{~Hz}, 2 \mathrm{H}$, $\mathrm{CH}_{2} \mathrm{Ph}$ ), 4.67 and 4.54 ( $\mathrm{AB}$ syst, $\left.J=11.5 \mathrm{~Hz}, 2 \mathrm{H}, \mathrm{CH}_{2} \mathrm{Ph}\right), 4.54$ and 4.43 (AB syst, $J=11.7,2 \mathrm{H},\left(\mathrm{H}_{2} \mathrm{Ph}\right), 4.32-4.27(\mathrm{~m}, 1 \mathrm{H}, \mathrm{H}-5), 4.14-4.08$ $\left(\mathrm{m}, 1 \mathrm{H}, \mathrm{H}-\mathrm{l}^{\prime}\right), 4.07-3.91\left(\mathrm{~m}, 5 \mathrm{H}, \mathrm{H}-1, \mathrm{H}-2, \mathrm{H}-3, \mathrm{H}-4, \mathrm{H}-3^{\prime}\right), 3.72-3.57$ $(\mathrm{m}, 2 \mathrm{H}, \mathrm{H}-6), 2.69\left(\mathrm{~s}, 3 \mathrm{H}, \mathrm{N}-\mathrm{CH}_{3}\right), 2.56-2.49\left(\mathrm{~m}, 1 \mathrm{H}, \mathrm{H}-2^{\prime}\right), 2.49-2.39$ $\left(\mathrm{m}, 1 \mathrm{H}, \mathrm{H}-2^{\prime}\right), 2.09-1.96(\mathrm{~m}, 2 \mathrm{H}), 1.80-1.64(\mathrm{~m}, 2 \mathrm{H}), 1.60-1.52(\mathrm{~m}$, 1H), $1.42-1.34(\mathrm{~m}, 1 \mathrm{H}), 1.32-1.25(\mathrm{~m}, 1 \mathrm{H}), 1.12(\mathrm{t}, J=12.3 \mathrm{~Hz}, 1 \mathrm{H})$, $0.92-0.79(\mathrm{~m}, 10 \mathrm{H}) .{ }^{13} \mathrm{C} \mathrm{NMR}\left(100.6 \mathrm{MHz} \mathrm{CDCl}_{3}\right): \delta=172.8(\mathrm{C}=\mathrm{O})$, 138.6 (C), 138.5 (C), 138.2 (C), 138.0 (C), $128.3(2 \mathrm{CH}), 128.2(\mathrm{CH})$, $128.1(\mathrm{CH}), 128.0(\mathrm{CH}), 127.8(\mathrm{CH}), 127.6(2 \mathrm{CH}), 127.5(\mathrm{CH}), 127.4(2$ $(\mathrm{H}), 127.2(\mathrm{CH}), 89.9(\mathrm{C}), 76.7\left(\mathrm{Cl}^{\prime}\right), 76.0,74.2,74.1,73.8,73.8,73.2$, $72.9,71.9,68.5(\mathrm{C} 6), 65.8\left(\mathrm{C}^{\prime}\right), 48.1(\mathrm{CH}), 40.0\left(\mathrm{CH}_{2}\right), 34.8\left(\mathrm{C}^{\prime}\right), 34.6$ $\left(\mathrm{CH}_{2}\right), 29.6(\mathrm{CH}), 25.9\left(\mathrm{CH}_{3}-\mathrm{N}\right), 24.3(\mathrm{CH}), 24.1\left(\mathrm{CH}_{3}\right), 22.3\left(\mathrm{CH}_{2}\right), 22.1$ $\left(\mathrm{CH}_{3}\right), 18.3\left(\mathrm{CH}_{3}\right)$.

Cycloadduct (11): Following the general procedure (4 h), a-C-vinylglucoside (289 mg, $0.52 \mathrm{mmol}$ ) afforded cycloadduct 11 (228 mg, $0.28 \mathrm{mmol}, 61 \%$ ) after purification by column chromatography (cyclohexane/EtOAC, 95:5 to 87:13). Colorless oil. $R_{f}=0.44$ (cyclohexane/EtOAC, 6:4). $(a]_{0}^{25}+48.4\left(\mathrm{C} 1.24, \mathrm{CHCl}_{3}\right)$. HRMS $\left.(\mathrm{ES})^{+}\right)$: Calculated for $\mathrm{C}_{49} \mathrm{H}_{61} \mathrm{~N}_{2} \mathrm{O}_{7}[\mathrm{M}+\mathrm{H}]^{+}: m / z=789.4473$, found 789.4453. ${ }^{1} \mathrm{H}$ NMR $\left(400 \mathrm{MHz}_{\mathrm{COCl}}\right): \delta 7.33-7.24(\mathrm{~m}, 18 \mathrm{H}, \mathrm{ArH}), 7.16-7.12(\mathrm{~m}, 2 \mathrm{H}, \mathrm{ArH})$, 4.91 and 4.78 ( $\mathrm{AB}$ syst, ) = $10.8 \mathrm{~Hz}, 2 \mathrm{H}, \mathrm{CH}_{2} \mathrm{Ph}$ ), 4.81 and 4.49 ( $\mathrm{AB}$ syst, $J=10.5 \mathrm{~Hz}, 2 \mathrm{H}, \mathrm{CH}_{2} \mathrm{Ph}$ ), 4.72 and 4.59 (AB syst, $J=11.6 \mathrm{~Hz}$, $2 \mathrm{H}, \mathrm{CH}_{2} \mathrm{Ph}$ ), 4.61 and $4.47\left(\mathrm{AB}\right.$ syst, $\left.J=12.0 \mathrm{~Hz}, 2 \mathrm{H}, \mathrm{CH}_{2} \mathrm{Ph}\right), 4.19-$ $\left.4.14\left(\mathrm{~m}, 1 \mathrm{H}, \mathrm{H}-\mathrm{\gamma}^{\prime}\right), 4.1\right)-4.06(\mathrm{~m}, \mathrm{lH}, \mathrm{H}-5), 3.98(\mathrm{t}, J=9.2 \mathrm{~Hz}, 1 \mathrm{H}, \mathrm{H}-$ 3), 3.96-3.88 (m, $\left.2 \mathrm{H}, \mathrm{H}-1, \mathrm{H}-3^{\prime}\right), 3.83-3.78(\mathrm{~m}, 1 \mathrm{H}, \mathrm{H}-2), 3.67-3.64(\mathrm{~m}$. $2 \mathrm{H}, \mathrm{H}-6), 3.61(\mathrm{t}, \mathrm{J}=9.2 \mathrm{~Hz}, 1 \mathrm{H}, \mathrm{H}-4), 2.72\left(\mathrm{~s}, 3 \mathrm{H}, \mathrm{N}-\mathrm{CH}_{3}\right), 2.69-2.51$ $\left(\mathrm{m}, 2 \mathrm{H}, \mathrm{H}-2^{\prime}\right), 2.16-2.01(\mathrm{~m}, 2 \mathrm{H}), 1.80-1.67(\mathrm{~m}, 2 \mathrm{H}), 1.59-1.50(\mathrm{~m}$,
$1 \mathrm{H}), 1.43-1.35(\mathrm{~m}, 1 \mathrm{H}), 1.33-1.25(\mathrm{~m}, 1 \mathrm{H}), 1.15(\mathrm{t}, J=12.3 \mathrm{~Hz}, 1 \mathrm{H})$ 0.93-0.72(m, 10H). ${ }^{13} \mathrm{C}$ NMR (100.6 $\left.\mathrm{MHz} \mathrm{CDCl}_{3}\right): \delta=173.0(\mathrm{C}=0)$, $138.9(\mathrm{C}), 138.4(\mathrm{C}), 138.0(\mathrm{C}), 137.9(\mathrm{C}), 128.4(2 \mathrm{CH}), 128.3(2 \mathrm{CH})$ $128.0(\mathrm{CH}), 127.9(\mathrm{CH}), 127.8(2 \mathrm{CH}), 127.6(3 \mathrm{CH}), 127.5(\mathrm{CH}), 89.9$ (C), $83.7(\mathrm{C} 3), 79.5(\mathrm{C} 2), 78.1(\mathrm{C} 4), 75.6\left(\mathrm{CH}_{2}\right), 75.4(\mathrm{C}), 74.9\left(\mathrm{CH}_{2}\right)$ $74.3(\mathrm{CS}), 74.0\left(\mathrm{CH}_{2}\right), 73.5\left(\mathrm{CH}_{2}\right), 72.9(\mathrm{Cl}), 69.0(\mathrm{C} 6), 66.0\left(\mathrm{C}^{\prime}\right), 48.2$ $(\mathrm{CH}), 40.2\left(\mathrm{CH}_{2}\right), 34.8\left(\mathrm{C}^{\prime}\right), 34.6\left(\mathrm{CH}_{2}\right), 29.3(\mathrm{CH}), 26.1\left(\mathrm{CH}_{3}\right), 24.4$ $(\mathrm{CH}), 24.1\left(\mathrm{CH}_{3}\right), 22.5\left(\mathrm{CH}_{3}\right), 22.1\left(\mathrm{CH}_{2}\right), 18.3\left(\mathrm{CH}_{3}\right)$.

Cycloadduct (12): Following the general procedure ( $4 \mathrm{~h})$, a-C-vinylmannoside (625 mg, $1.13 \mathrm{mmol}$ ) afforded cycloadduct 12 (561 mg, $0.71 \mathrm{mmol}, 69 \%$ ) after purification by column chromatography (cy. clohexane/EtOAC, $95: 5$ to $87: 13$ ). Colorless oil. $R_{f}=0.44$ (cyclohexane/EtOAC, 6:4). $[\alpha]_{D}^{25}+39.5$ (c $\left.1.00, \mathrm{CHCl}_{3}\right)$. HRMS $\left.(E S)^{+}\right)$: Calculated for $\mathrm{C}_{49} \mathrm{H}_{6}, \mathrm{~N}_{2} \mathrm{O},\left[\mathrm{M}+\mathrm{H}^{*}: \mathrm{m} / z=789.4473\right.$, found 789.4452. ${ }^{1} \mathrm{H}$ NMR (400 MHz, CDCl 3 ): $\delta 7.32-7.24(\mathrm{~m}, 18 \mathrm{H}, \mathrm{ArH}), 7.21-7.17(\mathrm{~m}, 2 \mathrm{H}, \mathrm{ArH})$, $4.69-4.56\left(\mathrm{~m}, 4 \mathrm{H}, \mathrm{CH}_{2} \mathrm{Ph}\right), 4.55-4.49\left(\mathrm{~m}, 4 \mathrm{H}, \mathrm{CH}_{2} \mathrm{Ph}\right), 4.03-3.96(\mathrm{~m}$, $\left.2 \mathrm{H}, \mathrm{H}-\mathrm{T}^{\prime}\right), 3.94-3.87\left(\mathrm{~m}, 3 \mathrm{H}, \mathrm{H}-1, \mathrm{H}-5, \mathrm{H}-3^{\prime}\right), 3.80-3.55(\mathrm{~m}, 4 \mathrm{H}, \mathrm{H}-2$ $\mathrm{H}-3, \mathrm{H}-6), 2.70\left(\mathrm{~s}, 3 \mathrm{H}, \mathrm{N}-\mathrm{CH}_{3}\right), 2.44-2.28\left(\mathrm{~m}, 2 \mathrm{H}, \mathrm{H}-2^{\prime}\right), 2.15-2.05(\mathrm{~m}$, IH), $1.95-1.88(m, 1 H), 1.80-1.66(m, 2 H), 1.58-1.51(m, 1 H), 1.41-$ $1.24(\mathrm{~m}, 2 \mathrm{H}), 1.19(\mathrm{t})=,12.4 \mathrm{~Hz}, 1 \mathrm{H}), 0.85-0.72(\mathrm{~m}, 10 \mathrm{H}) \cdot{ }^{13} \mathrm{C}$ NMR (100.6 $\left.\mathrm{MHz}, \mathrm{CDCl}_{3}\right): \delta=172.8(\mathrm{C}=0), 138.4(\mathrm{C}), 138.3(\mathrm{C}), 138.1$ (C), $137.9(\mathrm{C}), 128.4(2 \mathrm{CH}), 128.3(2 \mathrm{CH}), 128.2(\mathrm{CH}), 127.8(2 \mathrm{CH}), 127.7$ $(3 \mathrm{CH}), 127.6(\mathrm{CH}), 127.4(\mathrm{CH}), 89.6(\mathrm{C}), 77.9,74.9,74.8,74.6,74.3$, $73.7,73.4,73.2,72.4,71.6,69.2(\mathrm{C} 6), 66.0\left(\mathrm{C}^{\prime}\right), 48.2(\mathrm{CH}), 40.6\left(\mathrm{CH}_{2}\right)$, $34.6\left(\mathrm{CH}_{2}\right), 29.3(\mathrm{CH}), 26.9(\mathrm{CH}), 26.1\left(\mathrm{CH}_{3}-\mathrm{N}\right), 24.3(\mathrm{CH}), 24.1\left(\mathrm{CH}_{3}\right)$, $22.4\left(\mathrm{CH}_{3}\right), 22.2\left(\mathrm{CH}_{3}\right), 18.3\left(\mathrm{CH}_{3}\right)$.

Cycloadduct (13): Following the general procedure ( $8 \mathrm{~h}$ ), $\alpha$-C-vinyl$\mathrm{N}$-acetylgalactoside $(565 \mathrm{mg}, 1.13 \mathrm{mmol}$ ) afforded cycloadduct 13 $(580 \mathrm{mg}, 0.784 \mathrm{mmol}, 77 \%$ ) after purification by column chromatography (cyclohexane/EtOAc, 5:5 to 2:8). White solid. M.p. 71.5$73^{\circ} \mathrm{C} . R_{f}=0.2$ (cyclohexane/EtOAc, 3:7). $[\alpha]^{25}+22.6$ (c $1.0, \mathrm{CHCl}_{3}$ ). HRMS $\left(E S I^{+}\right)$: Calculated for $\mathrm{C}_{44} \mathrm{H}_{57} \mathrm{~N}_{3} \mathrm{O}_{7}\left[\mathrm{M}+\mathrm{H}^{+}: \mathrm{m} / \mathrm{z}=740.4269\right.$, found 740.4261. 'H NMR $\left(400 \mathrm{MHz}^{\circ} \mathrm{CDCl}_{3}\right): \delta 7.36-7.21(\mathrm{~m}, 15 \mathrm{H}$, ArH), $5.81(\mathrm{~d}, J=6.6 \mathrm{~Hz}, 1 \mathrm{H}, \mathrm{NH}), 4.74$ and 4.58 (AB syst, $f=11.8 \mathrm{~Hz}$, $2 \mathrm{H},\left(\mathrm{H}_{2} \mathrm{Ph}\right.$ ), 4.57 and 4.51 ( $\mathrm{AB}$ syst, $J=11.9 \mathrm{~Hz}, 2 \mathrm{H},\left(\mathrm{H}_{2} \mathrm{Ph}\right.$ ), 4.56 and 4.45 ( $\mathrm{AB}$ syst, $\left.)=11.9 \mathrm{~Hz}, 2 \mathrm{H}, \mathrm{CH}_{2} \mathrm{Ph}\right), 4.35-4.30(\mathrm{~m}, 1 \mathrm{H}, \mathrm{H}-5)$, 4.21-4.13 (m, 2H, H-1, H-2), 4.05-3.98 (m, 2H, H-3, H-6), 3.93-3.89 $\left(\mathrm{m}, \mathrm{H}, \mathrm{H}-3^{\prime}\right), 3.84-3.73\left(\mathrm{~m}, 3 \mathrm{H}, \mathrm{H}-1^{\prime}, \mathrm{H}-4, \mathrm{H}-6\right), 2.71\left(5,3 \mathrm{H}, \mathrm{N}-\mathrm{CH}_{3}\right)$ 2.54-2.40(m, 2H, H-2') $2.01-1.88(\mathrm{~m}, 2 \mathrm{H}), 1.90\left(\mathrm{~s}, 3 \mathrm{H}, \mathrm{COCH}_{3}\right), 1.82-$ $1.65(\mathrm{~m}, 3 \mathrm{H}), 1.62-1.54(\mathrm{~m}, 1 \mathrm{H}), 1.44-1.20(\mathrm{~m}, 3 \mathrm{H}), 0.88-0.80(\mathrm{~m}, 9 \mathrm{H})$. ${ }^{13} \mathrm{C}$ NMR (100.6 MHz, $\left.\mathrm{CDCl}_{3}\right): \delta=172.5(\mathrm{C}=0), 169.7(\mathrm{C}=0), 138.4$ (C), $138.1(\mathrm{C}), 137.7(\mathrm{C}), 128.3(3 \mathrm{CH}), 127.7(\mathrm{CH}), 127.6(3 \mathrm{CH}), 127.5$ $(\mathrm{CH}), 127.4(\mathrm{CH}), 89.9(\mathrm{C}), 77.6\left(\mathrm{C}^{\prime}\right), 75.5(\mathrm{Cs}), 74.6(\mathrm{C} 3), 73.1\left(\mathrm{CH}_{2}\right)$, $72.8\left(\mathrm{CH}_{2}\right), 71.9(\mathrm{C} 4), 77.5\left(\mathrm{CH}_{2}\right), 67.0(\mathrm{C} 1), 66.1(\mathrm{C} 6), 65.5\left(\mathrm{C}^{\prime}\right), 50.6$ $(\mathrm{C} 2), 48.1(\mathrm{CH}), 40.5\left(\mathrm{CH}_{2}\right), 34.9\left(\mathrm{C}^{\prime}\right), 34.6\left(\mathrm{CH}_{2}\right), 29.5(\mathrm{CH}), 26.0$ $\left(\mathrm{N}-\mathrm{CH}_{3}\right), 24.3(\mathrm{CH}), 24.1\left(\mathrm{CH}_{3}\right), 23.3\left(\mathrm{COCH}_{3}\right), 22.6\left(\mathrm{CH}_{3}\right), 22.1\left(\mathrm{CH}_{2}\right)$, $18.3\left(\mathrm{CH}_{3}\right)$

Cycloadduct (14): Following the general procedure ( $8 \mathrm{~h}), \beta$ - $C$-vinyl. $\mathrm{N}$-acetylgalactoside $(462 \mathrm{mg}, 0.921 \mathrm{mmol})$ afforded cycloadduct 14 (431 $\mathrm{mg}, 0.58 \mathrm{mmol}, 63 \%$ ) after purification by column chromatography (cyclohexane/EtOAC, 5:5 to 3:7). Orange solid. M.p. 69.5$71.5^{\circ} \mathrm{C} . R_{\mathrm{f}}=0.2$ (cyclohexane/EtOAC, 3:7). $[a]_{D}^{25}+40.1$ (c 1.0, $\mathrm{CHCl}_{3}$ ). HRMS $\left(E S I^{+}\right)$: Calculated for $\mathrm{C}_{44} \mathrm{H}_{58} \mathrm{~N}_{3} \mathrm{O} 7\left[\mathrm{M}+\mathrm{H}^{+}\right]^{+}: \mathrm{m} / z=740.4269$, found 740.4264. 'H NMR (400 MHz, COCl 3 ): $\delta 7.38-7.21(\mathrm{~m}, 15 \mathrm{H}$, ArH), 5.21 (d, $J=7.7 \mathrm{~Hz}, 1 \mathrm{H}, \mathrm{NH}$ ), 4.87 and 4.57 (AB syst, $J=11.6 \mathrm{~Hz}$, $2 \mathrm{H},\left(\mathrm{H}_{2} \mathrm{Ph}\right.$ ), 4.66 and 4.41 ( $\mathrm{AB}$ syst, $J=11.8 \mathrm{~Hz}, 2 \mathrm{H},\left(\mathrm{H}_{2} \mathrm{Ph}\right), 4.46$ and 4.41 ( $\mathrm{AB}$ syst, $\left.J=11.8 \mathrm{~Hz}, 2 \mathrm{H}, \mathrm{CH}_{2} \mathrm{Ph}\right), 4.01-3.97(\mathrm{~m}, 1 \mathrm{H}, \mathrm{H}-4)$, 3.95-3.84 (m, 3H, H-1', H-3', H-3), 3.84-3.75 (m, 1H, H-2), 3.73-3.67 $(\mathrm{m}, 1 \mathrm{H}, \mathrm{H}-1), 3.57-3.52(\mathrm{~m}, 3 \mathrm{H}, \mathrm{H}-5, \mathrm{H}-6), 2.68\left(\mathrm{~s}, 3 \mathrm{H}, \mathrm{N}-\mathrm{CH}_{3}\right), 2.71-$ $2.64\left(\mathrm{~m}, 1 \mathrm{H}, \mathrm{H}-2^{\prime}\right), 2.57-2.48\left(\mathrm{~m}, 1 \mathrm{H}, \mathrm{H}-2^{\prime}\right), 1.98-1.89(\mathrm{~m}, 2 \mathrm{H}), 1.86(\mathrm{~s}$, $\left.3 \mathrm{H}, \mathrm{COCH}_{3}\right), 1.82-1.74(\mathrm{~m}, 1 \mathrm{H}), 1.73-1.60(\mathrm{~m}, 1 \mathrm{H}), 1.59-1.50(\mathrm{~m}, 1 \mathrm{H})$, $1.42-1.33(\mathrm{~m}, 1 \mathrm{H}), 1.33-1.25(\mathrm{~m}, 1 \mathrm{H}), 1.25-1.16(\mathrm{~m}, 1 \mathrm{H}), 0.90-0.83$ 
$(\mathrm{m}, 1 \mathrm{H}), 0.90(\mathrm{~d}, J=6.4 \mathrm{~Hz}, 3 \mathrm{H}), 0.82(\mathrm{~d}, J=6.8 \mathrm{~Hz}, 3 \mathrm{H}), 0.79(\mathrm{~d}, J=$ $6.7 \mathrm{~Hz}, 3 \mathrm{H}) .{ }^{13} \mathrm{C} \mathrm{NMR}\left(100.6 \mathrm{MHz}, \mathrm{CDCl}_{3}\right): \delta=173.0(\mathrm{C}=0), 170.3(\mathrm{C}=$ $0), 138.6(\mathrm{C}), 138.0(\mathrm{C}), 137.9(\mathrm{C}), 128.5(\mathrm{CH}), 128.4(\mathrm{CH}), 128.2(\mathrm{CH})$, $128.0(2 \mathrm{CH}), 127.9(\mathrm{CH}), 127.8(\mathrm{CH}), 127.7(\mathrm{CH}), 127.4(\mathrm{CH}), 89.3(\mathrm{C})$, $79.4\left(\mathrm{Cl}^{\prime}\right.$ or $\left.\mathrm{C}^{\prime}\right), 78.1\left(\mathrm{Cl}^{\prime}\right.$ or $\left.\mathrm{C} 3^{\prime}\right), 77.2(\mathrm{C} 1), 76.5(\mathrm{C} 5), 74.3\left(\mathrm{CH}_{2}\right)$. $73.5\left(\mathrm{CH}_{2}\right), 72.3(\mathrm{C} 4), 71.5\left(\mathrm{CH}_{2}\right), 68.6(\mathrm{C} 6), 65.5(\mathrm{C} 3), 52.0(\mathrm{C} 2), 48.2$ $(\mathrm{CH}), 40.4\left(\mathrm{CH}_{2}\right), 35.0\left(\mathrm{Cl}^{\prime}\right), 34.7\left(\mathrm{CH}_{2}\right), 29.3(\mathrm{CH}), 26.0\left(\mathrm{~N}-\mathrm{CH}_{3}\right), 24.4$ $(\mathrm{CH}), 24.1\left(\mathrm{CH}_{3}\right), 23.7\left(\mathrm{COCH}_{3}\right), 22.6\left(\mathrm{CH}_{3}\right), 22.2\left(\mathrm{CH}_{2}\right), 18.4\left(\mathrm{CH}_{3}\right)$

General Procedure for Cleavage of Chiral Auxiliary: A solution of cycloadduct (7b and 8-14) in $\mathrm{ACOH}(12.5 \mathrm{~mL}$ for $1 \mathrm{mmol}$ ) and aqueous $1 \mathrm{M} \mathrm{HCl}(2.5 \mathrm{~mL}$ for $1 \mathrm{mmol})$ was stirred at $80^{\circ} \mathrm{C}$ for $2 \mathrm{~h}$. The reaction mixture was diluted with EtOAc $(30 \mathrm{~mL})$ and a saturated aqueous $\mathrm{NaHCO}_{3}$ solution $(30 \mathrm{~mL}$ ). Aqueous layer was extracted with ETOAc ( $30 \mathrm{~mL}$ ) and organic layers were dried with $\mathrm{MgSO}_{4}$, and concentrated under reduced pressure to afford the crude product.

$N$-Methyl-5-(2,3,4,6-tetra-O-benzyl- $\beta$-C-o-galactopyranos-1-yl)isoxazolidine-3-amide (15): The crude product was purified by column chromatography (cyclohexane/EtOAC, 80:20 to 25:75) to provide compound 15 (218 mg, $0.33 \mathrm{mmol}, 67 \%)$. Colorless oil. $R_{\mathrm{f}}=$

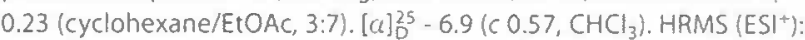
Calculated for $\mathrm{C}_{39} \mathrm{H}_{45} \mathrm{~N}_{2} \mathrm{O}_{7}[\mathrm{M}+\mathrm{H}]^{+}: \mathrm{m} / \mathrm{z}=653.3221$, found 653.3210. ${ }^{\top} \mathrm{H}$ NMR (400 $\left.\mathrm{MHz}_{2} \mathrm{CDCl}_{3}\right): \delta 7.38-7.23(\mathrm{~m}, 21 \mathrm{H}, \mathrm{ArH}, \mathrm{NH})$ 6.10-6.08 (m, 1H, NH), 4.98-4.85(m, 2H, $\left.\mathrm{CH}_{2} \mathrm{Ph}\right), 4.78-4.62(\mathrm{~m}, 2 \mathrm{H}$, $\left.\mathrm{CH}_{2} \mathrm{Ph}\right), 4.59-4.35\left(\mathrm{~m}, 5 \mathrm{H}, \mathrm{CH}_{2} \mathrm{Ph}, \mathrm{H}-1\right), 3.94-3.90(\mathrm{~m}, \mathrm{H}), 3.87-3.81$ $\left(\mathrm{m}, \mathrm{H}, \mathrm{H}-3^{\prime}\right), 3.65-3.54(\mathrm{~m}, 4 \mathrm{H}), 3.51-3.40(\mathrm{~m}, 2 \mathrm{H}), 2.79(\mathrm{~d}, \mathrm{~J}=4.9 \mathrm{~Hz}$, $\left.3 \mathrm{H}, \mathrm{NCH}_{3}\right), 2.64-2.56\left(\mathrm{~m}, 1 \mathrm{H}, \mathrm{H}-2^{\prime}\right), 2.42-2.34\left(\mathrm{~m}, 1 \mathrm{H}, \mathrm{H}-2^{\prime}\right) .{ }^{13} \mathrm{C}$ NMR (100.6 MHz, CDCl $): \delta=172.5$ (C=0), 138.6 (C), 138.0 (C), 137.6 (2 C), $128.5(\mathrm{CH}), 128.4(2 \mathrm{CH}), 128.2(2 \mathrm{CH}), 128.0(\mathrm{CH}), 127.9(2 \mathrm{CH})$, $127.8(2 \mathrm{CH}), 127.7(\mathrm{CH}), 127.4(\mathrm{CH}), 84.4,82.3,78.3,76.3,75.2\left(\mathrm{CH}_{2}\right)$, $75.1,74.4\left(\mathrm{CH}_{2}\right), 73.4\left(\mathrm{CH}_{2}\right), 72.2\left(\mathrm{CH}_{2}\right), 68.8(\mathrm{C} 6), 62.9\left(\mathrm{C}^{\prime}\right), 32.0$ (C2'), $25.8\left(\mathrm{CH}_{3}\right)$

$N$-Methyl-5-(2,3,4,6-tetra-O-benzyl- $\beta$-C-D-glucopyranos-1-yl)isoxazolidine-3-amide (16): The crude product was purified by column chromatography (cyclohexane/EtOAC, 80:20 to 25:75) to provide compound $16(94 \mathrm{mg}, 0.14 \mathrm{mmol}, 61 \%)$. White solid. M.p. $156.5-158.1^{\circ} \mathrm{C} . R_{f}=0.23$ (cyclohexane/EtOAC, 3:7). [a] $]_{D}^{25}-3.2$ (c 0.95, $\left.\mathrm{CHCl}_{3}\right)$. HRMS $\left(\mathrm{ESI}^{+}\right)$: Calculated for $\mathrm{C}_{39} \mathrm{H}_{45} \mathrm{~N}_{2} \mathrm{O}_{7}\left[\mathrm{M}+\mathrm{H}^{+}: \mathrm{m} / \mathrm{z}=\right.$ 653.3221, found 653.3233. ' $\mathrm{H}$ NMR (400 $\left.\mathrm{MHz}_{2} \mathrm{CDCl}_{3}\right)$ : o 7.36-7.18 $\left.\left(\mathrm{m}, 21 \mathrm{H}, \mathrm{NH}_{1} \mathrm{ArH}\right), 6.17(\mathrm{~d})=5.0,,1 \mathrm{H}, \mathrm{NH}\right), 4.91$ and 4.87 (AB syst. $J=10.9 \mathrm{~Hz}, 2 \mathrm{H},\left(\mathrm{H}_{2} \mathrm{Ph}\right), 4.83$ and 4.57 (AB syst. $J=11.0 \mathrm{~Hz}, 2 \mathrm{H}$, $\left(\mathrm{H}_{2} \mathrm{Ph}\right.$ ), 4.82 and 4.53 ( $\mathrm{AB}$ syst. $)=10.8 \mathrm{~Hz}, 2 \mathrm{H},\left(\mathrm{H}_{2} \mathrm{Ph}\right.$ ), 4.48 and 4.43 (AB syst. $\left.)=12.1 \mathrm{~Hz}, 2 \mathrm{H}, \mathrm{CH}_{2} \mathrm{Ph}\right), 4.41-4.36\left(\mathrm{~m}, 1 \mathrm{H}, \mathrm{H}-1^{\prime}\right), 3.85-$ $3.79\left(\mathrm{~m}, 1 \mathrm{H}, \mathrm{H}-3^{\prime}\right), 3.72-3.57(\mathrm{~m}, 3 \mathrm{H}, \mathrm{H}-1, \mathrm{H}-3, \mathrm{H}-6), 3.57-3.45(\mathrm{~m}$, $3 \mathrm{H}, \mathrm{H}-4, \mathrm{H}-5, \mathrm{H}-6), 3.21(\mathrm{t}, J=9.4 \mathrm{~Hz}, 1 \mathrm{H}, \mathrm{H}-2), 2.80(\mathrm{~d}, J=5.0 \mathrm{~Hz}$, $\left.3 \mathrm{H}, \mathrm{N}-\mathrm{CH}_{3}\right), 2.46-2.54\left(\mathrm{~m}, 1 \mathrm{H}, \mathrm{H}-2^{\prime}\right), 2.32-2.40\left(\mathrm{~m}, 1 \mathrm{H}, \mathrm{H}-2^{\prime}\right) .{ }^{13} \mathrm{C}$ NMR $\left(100.6 \mathrm{MHz}, \mathrm{COCl}_{3}\right): \delta=172.4(\mathrm{C}=0), 138.2(\mathrm{C}), 137.9(2 \mathrm{C}), 137.3$ (C), $128.6(\mathrm{CH}), 128.5(3 \mathrm{CH}), 128.4(\mathrm{CH}), 128.2(\mathrm{CH}), 127.9(2 \mathrm{CH})$, $127.8(\mathrm{CH}), 127.7(2 \mathrm{CH}), 127.6(\mathrm{CH}), 86.9(\mathrm{C} 3), 82.0(\mathrm{C} 1), 78.7(\mathrm{C} 2)$, 78.3 (C4 or $\mathrm{C} 5), 78.2\left(\mathrm{C}^{\prime}\right), 78.0(\mathrm{C} 4$ or $\mathrm{C5}), 75.5\left(\mathrm{CH}_{2}\right), 75.1\left(\mathrm{CH}_{2}\right)$, $75.0\left(\mathrm{CH}_{2}\right), 73.2\left(\mathrm{CH}_{2}\right), 69.1(\mathrm{C} 6), 62.8\left(\mathrm{C}^{\prime}\right), 32.0\left(\mathrm{C}^{\prime}\right), 25.9\left(\mathrm{CH}_{3}\right)$.

N-Methyl-5-(2,3,4,6-tetra-O-benzyl- $\beta$-C-D-mannopyranos-1-yl)isoxazolidine-3-amide (17): The crude product was purified by column chromatography (cyclohexane/EtOAC, 80:20 to 25:75) to provide compound 17 (210 mg, $0.32 \mathrm{mmol}, 59 \%)$. Colorless oil. $R_{\mathrm{f}}=$ 0.25 (cyclohexane/EtOAC, 3:7). [ $\alpha]_{0}^{25}+2.3$ (c 1.03, $\mathrm{CHCl}_{3}$ ). HRMS (ESI ${ }^{+}$): Calculated for $\mathrm{C}_{39} \mathrm{H}_{45} \mathrm{~N}_{2} \mathrm{O}_{7}[\mathrm{M}+\mathrm{H}]^{+}: \mathrm{m} / 2=653.3221$, found 653.3232. ' $\mathrm{H}$ NMR $\left(400 \mathrm{MHz}, \mathrm{CDCl}_{3}\right): \delta 7.37-7.24(\mathrm{~m}, 19 \mathrm{H}, \mathrm{ArH}, \mathrm{NH})$, 7.22-7.18 ( $\mathrm{m}, 2 \mathrm{H}, \mathrm{ArH}), 6.09-5.86(\mathrm{br}, 1 \mathrm{H}, \mathrm{NH}), 4.93$ and 4.63 ( $\mathrm{AB}$ Syst. $J=11.3 \mathrm{~Hz}, 2 \mathrm{H}, \mathrm{CH}_{2} \mathrm{Ph}$ ), 4.84 and 4.56 (AB syst. $J=10.8 \mathrm{~Hz}$, $2 \mathrm{H}, \mathrm{CH}_{2} \mathrm{Ph}$ ), 4.75 and 4.70 ( $\mathrm{AB}$ syst. $)=11.8 \mathrm{~Hz}, 2 \mathrm{H}, \mathrm{CH}_{2} \mathrm{Ph}$ ) 4.57 and 4.47 ( $\mathrm{AB}$ syst. $\left.J=12.0 \mathrm{~Hz}, 2 \mathrm{H}, \mathrm{CH}_{2} \mathrm{Ph}\right), 4.19-4.13\left(\mathrm{~m}, 1 \mathrm{H}, \mathrm{H}-1^{\prime}\right.$ ), $3.92-3.90(\mathrm{~m}, 1 \mathrm{H}, \mathrm{H}-2), 3.86-3.81(\mathrm{~m}, 2 \mathrm{H}, \mathrm{H}-3, \mathrm{H}-4), 3.72-3.67(\mathrm{~m}$, $1 \mathrm{H}, \mathrm{H}-6), 3.65-3.56(\mathrm{~m}, 2 \mathrm{H}, \mathrm{H}-3, \mathrm{H}-6), 3.50-3.45(\mathrm{~m}, \mathrm{IH}, \mathrm{H}-5), 3.43-$ $3.40(\mathrm{~m}, 1 \mathrm{H}, \mathrm{H}-1), 2.92-2.84\left(\mathrm{~m}, 1 \mathrm{H}, \mathrm{H}-2^{\prime}\right), 2.80(\mathrm{~d}, J=5.0 \mathrm{z}, 3 \mathrm{H}, \mathrm{N}-$ $\left.\mathrm{CH}_{3}\right), 2.70-2.62\left(\mathrm{~m}, 1 \mathrm{H}, \mathrm{H}-2^{\prime}\right),{ }^{13} \mathrm{C} \mathrm{NMR}\left(100.6 \mathrm{MHz}_{2}, \mathrm{CDCl}_{3}\right): \delta=172.2$ $(\mathrm{C}=0), 138.1(4 \mathrm{C}), 128.4(\mathrm{CH}), 128.3(3 \mathrm{CH}), 128.2(\mathrm{CH}), 128.0(2 \mathrm{CH})$, $127.7(2 \mathrm{CH}), 127.6(\mathrm{CH}), 127.5(\mathrm{CH}), 127.4(\mathrm{CH}), 84.5(\mathrm{C} 3), 80.2(\mathrm{Cl})$, $79.5\left(\mathrm{Cl}^{\prime}\right.$ and $\left.\mathrm{C5}\right), 75.2\left(\mathrm{CH}_{2}\right), 75.0(\mathrm{C} 4), 74.4\left(\mathrm{CH}_{2}\right), 74.4(\mathrm{C} 2), 73.2$ $\left(\mathrm{CH}_{2}\right), 72.4\left(\mathrm{CH}_{2}\right), 69.6\left(\mathrm{C}^{2}\right), 62.6\left(\mathrm{C}^{\prime}\right), 34.8\left(\mathrm{C}^{\prime}\right), 25.8\left(\mathrm{CH}_{3}\right)$.

N-Methyl-5-\{2,3,4,6-tetra-O-benzyl- $a-C-0$-galactopyranos-1-yl\}isoxazolidine-3-amide (18): The crude product was purified by Column chromatography (cyclohexane/EtOAC, 80:20 to 25:75) to provide compound $18(809 \mathrm{mg}, 1.24 \mathrm{mmol}, 62 \%)$. Colorless oil. $R_{f}=$ 0.13 (cyclohexane/EtOAC, 3:7). [u $]_{0}^{25}+46.7$ (c $0.55, \mathrm{CHCl}_{3}$ ). HRMS $\left(\right.$ ESI ${ }^{+}$): Calculated for $\mathrm{C}_{39} \mathrm{H}_{44} \mathrm{~N}_{2} \mathrm{O}_{7} \mathrm{Na}[\mathrm{M}+\mathrm{Na}]^{+}: \mathrm{m} / 2=675.3041$, found 675.3043. 'H NMR $\left(400 \mathrm{MHz}_{1} \mathrm{CDCl}_{3}\right): \delta 7.36-7.21(\mathrm{~m}, 21 \mathrm{H}$, ArH, NH), 4.73-4.46 (m, 8H, $\left.\mathrm{CH}_{2} \mathrm{Ph}\right), 4.39-4.32\left(\mathrm{~m}, 1 \mathrm{H}, \mathrm{H} \cdot \mathrm{1}^{\prime}\right), 4.31-$ $4.25(\mathrm{~m}, 1 \mathrm{H}, \mathrm{H}-1), 4.02-3.96(\mathrm{~m}, 1 \mathrm{H}), 3.91-3.64(\mathrm{~m}, 5 \mathrm{H}), 3.61-3.50(\mathrm{~m})$ $1 \mathrm{H}, \mathrm{H} \cdot 6), 2.80-2.74\left(\mathrm{~m}, 3 \mathrm{H}, \mathrm{N} \cdot \mathrm{CH}_{3}\right), 2.70-2.60\left(\mathrm{~m}, 1 \mathrm{H}, \mathrm{H} \cdot 2^{\prime}\right), 2.28-$ $2.19\left(\mathrm{~m}, 1 \mathrm{H}, \mathrm{H}-2^{\prime}\right) .{ }^{13} \mathrm{C}$ NMR $\left(100.6 \mathrm{MHz}, \mathrm{COCl}_{3}\right): \delta 171.7(\mathrm{C}=\mathrm{O}), 138.4$ (C) $138.3(\mathrm{C}), 138.2(\mathrm{C}), 137.8(\mathrm{C}), 128.4(3 \mathrm{CH}), 128.3(\mathrm{CH}), 128.1$ $(\mathrm{CH}), 127.9(\mathrm{CH}), 127.8(2 \mathrm{CH}), 127.7(\mathrm{CH}), 127.6(2 \mathrm{CH}), 127.5(\mathrm{CH})$, $78.9(\mathrm{Cl}), 76.0(\mathrm{CH}), 74.7(\mathrm{C}), 74.0(\mathrm{CH}), 73.3\left(\mathrm{CH}_{2}\right), 73.2\left(3 \mathrm{CH}_{2}\right)$, $72.9(\mathrm{CH}), 71.3(\mathrm{CH}), 68.0(\mathrm{C} 6), 62.2\left(\mathrm{C}^{\prime}\right), 36.9\left(\mathrm{C}^{\prime}\right), 25.9\left(\mathrm{CH}_{3}\right)$.

N-Methyl-5-(2,3,4,6-tetra-O-benzyl-a-C-D-glucapyranos-1-yl)isoxazolidine-3-amide (19): The crude product was purified by column chromatography (cyclohexane/EtOAC, 80:20 to 25:75) to provide compound $19(211 \mathrm{mg}, 0.32 \mathrm{mmol}, 73 \%)$. Colorless oil. $R_{f}=$ 0.13 (cyclohexane/EtOAC, 3:7). [a] $]^{25}+34.2$ (c 1.04, $\mathrm{CHCl}_{3}$ ). HRMS $\left(\mathrm{ESI}^{+}\right)$: Calculated for $\mathrm{C}_{39} \mathrm{H}_{44} \mathrm{~N}_{2} \mathrm{O}_{7} \mathrm{Na}[\mathrm{M}+\mathrm{Na}]^{+}: \mathrm{m} / z=675.3041$ found 675.3041. ' $\mathrm{H}$ NMR $\left(400 \mathrm{MHz} \mathrm{CDCl}_{3}\right)$ : $\delta 7.37-7.24(\mathrm{~m}, 18 \mathrm{H}$, ArH) $7.23-7.18(\mathrm{~m}, 2 \mathrm{H}, \mathrm{ArH}), 7.12$ (br s, $1 \mathrm{H}, \mathrm{NH}), 5.96$ (br s, $1 \mathrm{H}, \mathrm{NH})$, 4.87 and 4.78 ( $\mathrm{AB}$ syst. $)=10.8 \mathrm{~Hz}, 2 \mathrm{H},\left(\mathrm{H}_{2} \mathrm{Ph}\right), 4.78$ and 4.74 ( $\mathrm{AB}$ syst. $)=11.8 \mathrm{~Hz}, 2 \mathrm{H},\left(\mathrm{H}_{2} \mathrm{Ph}\right.$ ), 4.60 and 4.56 ( $\mathrm{AB}$ syst. $)=12.0 \mathrm{~Hz}$, $\left.2 \mathrm{H}, \mathrm{CH}_{2} \mathrm{Ph}\right), 4,50-4.43\left(\mathrm{~m}, 3 \mathrm{H}, \mathrm{CH}_{2} \mathrm{Ph}, \mathrm{H}-\mathrm{I}^{\prime}\right)$ 4,02-3.85 (m, 3H, H-2, H4. $\mathrm{H}-3), 3.81-3.75(\mathrm{~m}, 2 \mathrm{H}, \mathrm{H}-1, \mathrm{H} \cdot 3), 3.68(\mathrm{dd}, 1 \mathrm{H})=10.4,,2.3 \mathrm{~Hz}$, $H-6), 3.56(\mathrm{dd}, 1 \mathrm{H})=10.4,,5.3 \mathrm{~Hz}, \mathrm{H}-6), 3.55-3.51(\mathrm{~m}, 1 \mathrm{H}, \mathrm{H}-5)$, $3.43-3.40(\mathrm{~m}, 1 \mathrm{H}, \mathrm{H} \cdot 1), 2.80\left(\mathrm{~d}, J=5.0 \mathrm{~Hz}, 3 \mathrm{H}, \mathrm{N}-\mathrm{CH}_{3}\right), 2.79-2.70(\mathrm{~m}$, $\left.1 \mathrm{H}, \mathrm{H}-2^{\prime}\right), 2.45-2.35\left(\mathrm{~m}, 1 \mathrm{H}, \mathrm{H}-2^{\prime}\right) .{ }^{13} \mathrm{C} \mathrm{NMR}\left(100.6 \mathrm{MHz} \mathrm{CDCl}_{3}\right)$ : d $171.3(\mathrm{C}=0), 138.5(\mathrm{C}), 138.1(\mathrm{C}), 138.0(\mathrm{C}), 137.7(\mathrm{C}), 128.5(\mathrm{CH})$, $128.4(\mathrm{CH}), 128.3(2 \mathrm{CH}), 128.0(\mathrm{CH}), 127.9(\mathrm{CH}), 127.8(3 \mathrm{CH}), 127.7$ $(2 \mathrm{CH}), 127.6(\mathrm{CH}), 82.4(\mathrm{C2}), 78.8(\mathrm{C} 1), 77.9(\mathrm{C}), 76.5(\mathrm{Cl}), 75.1$ $\left(\mathrm{CH}_{2}\right), 74.7(\mathrm{C} 4), 74.6\left(\mathrm{CH}_{2}\right), 73.7\left(\mathrm{CH}_{2}\right), 73.5\left(\mathrm{CH}_{2}\right), 69.6(\mathrm{C} 6), 62.2$ $\left(\mathrm{C}^{\prime}\right), 36.9\left(\mathrm{C2}^{\prime}\right), 26.0\left(\mathrm{CH}_{3}\right)$.

$N$-Methyl-5-(2,3,4,6-tetra-O-benzyl- $\alpha$-C-D-mannopyranos-1-yl)isoxazolidine-3-amide (20): The crude product was purified by column chromatography (cyclohexane/AcOEt, 80:20 to 25:75) to provide compound 20 (297 mg, $0.45 \mathrm{mmol}, 62 \%)$. Colorless oil. $R_{f}=$ 0.15 (cyclohexane/EtOAC, 3:7). [ $\alpha]_{0}^{25}+60.7$ (c 1.0, $\mathrm{CHCl}_{3}$ ). HRMS (ESI $)^{+}$): Caiculated for $\mathrm{C}_{39} \mathrm{H}_{45} \mathrm{~N}_{2} \mathrm{O},[\mathrm{M}+\mathrm{H}]^{+} ; \mathrm{m} / \mathrm{z}=653.3221$, found 653.3215. ${ }^{1} \mathrm{H}$ NMR $\left(400 \mathrm{MHz}_{2} \mathrm{CDCl}_{3}\right): \delta 7.36-7.18(\mathrm{~m}, 21 \mathrm{H}, \mathrm{NH}, \mathrm{ArH})$, 76.37 (br s, $1 \mathrm{H}, \mathrm{NH}), 4.58-4.42\left(\mathrm{~m}, 7 \mathrm{H}, \mathrm{CH}_{2} \mathrm{Ph}, \mathrm{H}-1\right.$ ) $), 4.40$ and 4.34 ( $\mathrm{AB}$ syst. $\left.)=12.2 \mathrm{~Hz}, 2 \mathrm{H}, \mathrm{CH}_{2} \mathrm{Ph}\right), 4.13-4.08(\mathrm{~m}, 1 \mathrm{H}, \mathrm{H}-5), 4.03$ (dd, $J=9.7,2.8 \mathrm{~Hz}, 1 \mathrm{H}, \mathrm{H}-3), 3.94-3.90\left(\mathrm{~m}, 1 \mathrm{H}, \mathrm{H}-3^{\prime}\right), 3.82(\mathrm{t}, J=2.8 \mathrm{~Hz}$, $1 \mathrm{H}, \mathrm{H}-2), 3.77(\mathrm{dd}, J=9.7,0.8 \mathrm{~Hz}, 1 \mathrm{H}, \mathrm{H}-4), 3.73(\mathrm{dd}, 1 \mathrm{H}, J=10.1$, $8.2 \mathrm{~Hz}, \mathrm{H}-6), 3.55-3.49(\mathrm{~m}, 2 \mathrm{H}, \mathrm{H}-1, \mathrm{H}-6), 2.84-2.77(\mathrm{~m}, 1 \mathrm{H}, \mathrm{H}-2$ ) , $\left.2.80(\mathrm{~d})=,5.0 \mathrm{~Hz}, 3 \mathrm{H}, \mathrm{N}-\mathrm{CH}_{3}\right), 2.64-2.56\left(\mathrm{~m}, 1 \mathrm{H}, \mathrm{H}-2^{\prime}\right) .{ }^{13} \mathrm{C} \mathrm{NMR}$ (100.6 $\left.\mathrm{MHz}_{2} \mathrm{CDCl}_{3}\right): \delta=172.7(\mathrm{C}=0), 137.9(2 \mathrm{C}), 137.8(2 \mathrm{C}), 128.4$ $(5 \mathrm{CH}), 127.9(3 \mathrm{CH}), 127.8(\mathrm{CH}), 127.7(2 \mathrm{CH}), 127.5(\mathrm{CH}), 77.3(\mathrm{Cl})$. $75.4(\mathrm{C} 1), 74.9(\mathrm{CS}), 74.1(\mathrm{C} 3), 73.6(\mathrm{C} 2), 73.3\left(\mathrm{CH}_{2}\right), 73.0(\mathrm{C} 4), 72.8$ $\left(\mathrm{CH}_{2}\right), 72.3\left(\mathrm{CH}_{2}\right), 71.4\left(\mathrm{CH}_{2}\right), 68.3\left(\mathrm{C}^{2}\right), 62.7\left(\mathrm{Cl}^{\prime}\right), 34.8\left(\mathrm{Cl}^{\prime}\right), 25.9$ $\left(\mathrm{CH}_{3}\right)$. 
N-Methyl-5-(2-acetamido-3,4,6-tri-O-benzyl- $\alpha$-C-D-galactopyranos-1-y|)-isoxazolidine-3-amide (21): The crude product was purified by column chromatography $\left(\mathrm{CH}_{2} \mathrm{Cl}_{2} / \mathrm{MeOH}, 98: 2 \rightarrow 96: 4\right)$ to provide compound 21 (268 $\mathrm{mg}, 0.444 \mathrm{mmol}, 58 \%$ ). White solid. M.p. $73-6-75^{\circ} \mathrm{C} . R_{\mathrm{f}}=0.15\left(\mathrm{CH}_{2} \mathrm{Cl}_{2} / \mathrm{MeOH}, 95: 5\right) \cdot[\mathrm{a}]_{\mathrm{D}}^{25}+51.8(\mathrm{c} 0.75$, $\left.\mathrm{CHCl}_{3}\right)$. HRMS (ESI): Calculated for $\mathrm{C}_{34} \mathrm{H}_{42} \mathrm{~N}_{3} \mathrm{O}_{7}(\mathrm{M}+\mathrm{H})^{+}: \mathrm{m} / \mathrm{z}=$ 604.3017, found 604.2985. 'H NMR (400 $\left.\mathrm{MHz}_{1} \mathrm{CDCl}_{3}\right): \delta$ 7.37-7.22 ( $\mathrm{m}, 15 \mathrm{H}, \mathrm{ArH}$ ), 7.07 (br s, 1H, NHMe), 6.62 (br s, 1H, NHAc), 6.09 (br s, $1 \mathrm{H}, \mathrm{NH}), 4.73$ and 4.68 ( $\mathrm{AB}$ syst. $J=11.8 \mathrm{~Hz}, 2 \mathrm{H}, \mathrm{CH}_{2} \mathrm{Ph}$ ), 4.55 and 4.47 ( $\mathrm{AB}$ syst. $J=11.8 \mathrm{~Hz}, 2 \mathrm{H}, \mathrm{CH}_{2} \mathrm{Ph}$ ), 4.51 and 4.41 ( $\mathrm{AB}$ syst. $J=$ $\left.12.0 \mathrm{~Hz}, 2 \mathrm{H}, \mathrm{CH}_{2} \mathrm{Ph}\right), 4.32-4.26(\mathrm{~m}, 1 \mathrm{H}, \mathrm{H}-5), 4.19-4.16(\mathrm{~m}, 1 \mathrm{H}, \mathrm{H}-3)$, $4.15(\mathrm{dd}, J=11.6,1.9 \mathrm{~Hz}, 1 \mathrm{H}, \mathrm{H}-6), 4.13-4.07\left(\mathrm{~m}, 1 \mathrm{H}, \mathrm{H}^{\prime} \mathbf{1}^{\prime}\right), 4.02-$ $3.88\left(\mathrm{~m}, 3 \mathrm{H}, \mathrm{H}-1, \mathrm{H}-2, \mathrm{H}-3^{\prime}\right), 3.78(\mathrm{dd}, J=6.5,2.9 \mathrm{~Hz}, 1 \mathrm{H}, \mathrm{H}-4), 3.69$ (dd, $J=11.6,2.1 \mathrm{~Hz}, 1 \mathrm{H}, \mathrm{H}-6), 2.99-2.87\left(\mathrm{~m}, 1 \mathrm{H}, \mathrm{H}-\mathbf{2}^{\prime}\right), 2.80(\mathrm{~d}, J=$ $5.0 \mathrm{~Hz}, 3 \mathrm{H}, \mathrm{CH}_{3}-\mathrm{N}$ ), 2.37 (ddd, $J=12.7,9.2,5.5 \mathrm{~Hz}_{1}, 1 \mathrm{H}, \mathrm{H}-2^{\prime}$ ), 1.98 (s, $\left.3 \mathrm{H}, \mathrm{CH}_{3}-\mathrm{CO}\right) .{ }^{13} \mathrm{C}$ NMR $\left(100.6 \mathrm{MHz}_{2} \mathrm{CDCl}_{3}\right): \delta 171.5(\mathrm{C}=0)$, $170.4(\mathrm{C}=0), 138.3(\mathrm{C}), 137.9(\mathrm{C}), 137.6(\mathrm{C}), 128.4(2 \mathrm{CH}), 128.2(\mathrm{CH})$, $128.0(\mathrm{CH}), 127.8(2 \mathrm{CH}), 127.6(2 \mathrm{CH}), 127.5(\mathrm{CH}), 79.6\left(\mathrm{Cl}^{\prime}\right), 75.0$ (C5), $73.3\left(\mathrm{CH}_{2}\right), 73.2(\mathrm{C} 3), 73.1\left(\mathrm{CH}_{2}\right), 72.4(\mathrm{C} 4), 70.9\left(\mathrm{CH}_{2}\right), 68.2(\mathrm{C} 1)$, $65.2(\mathrm{C} 6), 62.4\left(\mathrm{C}^{\prime}\right), 52.6(\mathrm{C} 2), 37.1\left(\mathrm{C} 2^{\prime}\right), 25.9\left(\mathrm{CH}_{3}-\mathrm{N}\right), 23.5$ $\left(\mathrm{CH}_{3}-\mathrm{CO}\right)$.

$N$-Methyl-5-(2-acetamido-3,4,6-tri-0-benzyl-B-C-0-galactopyranos-1-yl)-isoxazolidine-3-amide (22): The crude product was purified by column chromatography $\left(\mathrm{CH}_{2} \mathrm{Cl}_{2} / \mathrm{MeOH}, 98: 2 \rightarrow 96: 4\right)$ to provide compound 22 (178 $\mathrm{mg}, 0.295 \mathrm{mmol}, 55 \%$ ). Yellow solid. M.p. 58.5-61.2 ${ }^{\circ} \mathrm{C} . R_{f}=0.13\left(\mathrm{CH}_{2} \mathrm{Cl}_{2} / \mathrm{MeOH}, 95: 5\right) \cdot[a]_{\mathrm{D}}^{25}+31.9$ (c 0.7, $\left.\mathrm{CHCl}_{3}\right)$. HRMS $\left(\left.\mathrm{ESI}\right|^{+}\right)$: Calculated for $\mathrm{C}_{34} \mathrm{H}_{4} \mathrm{~N}_{3} \mathrm{NaO}_{7}[\mathrm{M}+\mathrm{Na}]^{+}: \mathrm{m} / \mathrm{z}=$ 626.2837, found 626.2816. ' $\mathrm{H}$ NMR (400 $\left.\mathrm{MHz} \mathrm{CDCl}_{3}\right)$ : $\delta$ 7.38-7.23 (m, 15H, ArH) 7.14 (br s, 1H, NHMe), 6.10 (br s, 1H, NHAc), 5.51 (d, $J=8.0 \mathrm{~Hz}, 1 \mathrm{H}, \mathrm{NH}), 4.90$ and 4.54 ( $\mathrm{AB}$ syst. $J=11.7 \mathrm{~Hz}, 2 \mathrm{H},\left(\mathrm{H}_{2} \mathrm{Ph}\right.$ ), 4.69 and 4.39 ( $\mathrm{AB}$ syst. $\left.J=11.9 \mathrm{~Hz}, 2 \mathrm{H}, \mathrm{CH}_{2} \mathrm{Ph}\right), 4.45$ and 4.41 ( $\mathrm{AB}$ syst. $\left.J=10.7 \mathrm{~Hz}, 2 \mathrm{H}, \mathrm{CH}_{2} \mathrm{Ph}\right), 4.25-4.18(\mathrm{~m}, 1 \mathrm{H}, \mathrm{HT}), 3.99-3.94(\mathrm{~m}$, $1 \mathrm{H}, \mathrm{H} 4), 3.91-3.81\left(\mathrm{~m}, 2 \mathrm{H}, \mathrm{Hz}, \mathrm{H}^{\prime}\right), 3.77(\mathrm{dd}, J=10.5,1.6 \mathrm{~Hz}, 1 \mathrm{H}$, $\mathrm{H} 1), 3.64-3.56(\mathrm{~m}, 2 \mathrm{H}, \mathrm{H} 3, \mathrm{HS}), 3.50(\mathrm{dd}, J=9.0,5.8 \mathrm{~Hz}, 1 \mathrm{H}, \mathrm{H}-6)$, $3.47(\mathrm{dd}, J=9.0,7.0 \mathrm{~Hz}, 1 \mathrm{H}, \mathrm{H}-6), 2.77\left(\mathrm{~d}, J=5.0 \mathrm{~Hz}, 3 \mathrm{H}, \mathrm{CH}_{3}-\mathrm{N}\right)$, 2.76-2.63 (m, 2H, H2') $1.86\left(\mathrm{~s}, 3 \mathrm{H}, \mathrm{CH}_{3}-\mathrm{CO}\right) .{ }^{13} \mathrm{C}$ NMR $(100.6 \mathrm{MHz}$, $\left(\mathrm{CDCl}_{3}\right): \delta=172.4(\mathrm{C}=0), 170.5(\mathrm{C}=0), 138.4(\mathrm{C}), 137.9(\mathrm{C}), 137.6(\mathrm{C})$, $128.5(\mathrm{CH}), 128.4(\mathrm{CH}), 128.2(\mathrm{CH}), 128.0(\mathrm{CH}), 127.9(\mathrm{CH}), 127.8(3$ $\mathrm{CH}), 127.5(\mathrm{CH}), 81.4(\mathrm{C} 1), 79.4(\mathrm{C} 5$ or $\mathrm{C} 3), 78.9\left(\mathrm{C} 1^{\prime}\right), 76.3(\mathrm{C} 3$ or $\mathrm{C} 5)$, $74.4\left(\mathrm{CH}_{2}\right), 73.4\left(\mathrm{CH}_{2}\right), 72.1(\mathrm{C} 4), 71.3\left(\mathrm{CH}_{2}\right), 68.9(\mathrm{C} 6), 62.8\left(\mathrm{C}^{\prime}\right), 49.3$ (C2), $32.6\left(\mathrm{Cl}^{\prime}\right), 25.8\left(\mathrm{CH}_{3}-\mathrm{N}\right), 23.3\left(\mathrm{CH}_{3}-\mathrm{CO}\right)$.

\section{Acknowledgments}

This work was supported by the Région des Pays de la Loire through the GlycoOuest program and the Ministere de l'Enseignement Supérieur et de la Recherche of France [Ph.D. fellowship (F. R.)]. We also thank Frédéric Legros for technical support, Sullivan Bricaud for the NMR analyses, Patricia Gangnery and Alexandre Bénard for the HRMS analyses.

\section{Keywords: Amino acids - C-glycosides - Cycloaddition} Nitrones. TN antigen analog

[1] R. A. Dwek, Chem. Rev. 1996, 96, 683-720

[2] T. Ju, V. I. Otto, R. D. Cummings, Angew. Chem. Int. Ed. 2011, 50, 17701791; Angew. Chem. 2011, 123,1808.

[3] For example, see: G. Yang, J. Schmieg, M. Tsuj\}, R. W. Franck, Angew. Chem. Int. Ed. 2004, 43, 3818-3822; Angew. Chem. 2004, 116. 3906.

(4) a) L. A. Marcaurelle, C. R. Bertozzi, Chem. Eur. J. 1999, 5, 1384-1390; b) A. Dondoni, A. Marra, Chem. Rev. 2000, 100,4395-4422; c) E. von Moos, R. N. Ben, Curr. Top. Med. Chem. 2005, 5, 1351-1361.
[5] D. Urban, T. Skrydstrup, J.-M. Beau, Chem. Commun. 1998, 955-956.

[6] a) A. Dondoni, A. Marra, A. Massi, Chem. Commun. 1998, 1741-1742; b) A. Dondoni, A. Marra, A. Massi, J. Org. Chem. 1999, 64, 933-944.

[7] a) A. Dondoni, A. Marra, P. P. Giovannini, /. Chem. Soc Perkin Trons. 2001, 2380-2388; b) E. Nolen. A. Kurish, J. Potter, L. Donahue, M. Orlando, Org. Lett. 2005, 7, 3383-3386; c) F. W. Schmidtmann, T. E. Benedum, G. J. McGarvey, Terrahedron Lett. 2005, 46, 4677-4681.

[8] 5. Koch, D. Schollmeyer, H. Lowe, H. Kunz, Chem. Eur. J. 2013, 19, 70207041.

[9] a) C. R. Bertozzi, P. D. Hoeprich, M. D. Bednarski, J. Org. Chem. 1992, 57, 6092-6094; b) T. Fuchs, R. R. Schmidt, Synthesis $1998,753-758$; c) S. D. Debenham, J. Cossrow, E. J. Toone, J. Org. Chem. 1999, 64, 9153-9163.

[10] A. D. Campbell, D. E. Paterson, T. M. Raynham, R. J. K. Taylor, Chem. Com. mun. 1999, 1599-1600.

[11] a) A. Nuzzi, A. Massi, A. Dondoni, Org. Lett. 2008, 10, 4485-4488; b) R. S Andrews, J. J. Becker, M. R. Gagné, Angew. Chem. Int. Ed. 2012, 51, 41404143; Angew. Chem. 2012, 124, 4216.

[12] a) T. Gustafsson, M. Saxin, J. Kihlberg, J. Org. Chem. 2003, 68, 2506-2509: b) N. Bragnier, R. Guillot, M.C. Scherrmann, Org. Biomol. Chem. 2009, ?, 3918-3921.

[13] A. J. Pearce, S. Ramaya, S. N. Thorn, G B. Bloomberg. D S. Walter, T. Gallagher, J. Org. Chem. 1999, 64, 5453-5462.

[14] J. L. Koviach, M. D. Chappell, R. L. Halcomb, J. Org. Chem, 2001, 66. 23182326.

[15] J. W. Lane, R. L. Hatcomb, J. Org. Chem. 2003, 68, 1348-1357.

[16] A. Dondoni, P. P. Giovannini, A. Massi, Org. Lett. 2004, 6, 2929-2932.

[17] A. Dondoni, A. Massi, M. Aldhoun, J. Org. Chem. 2007, 72, 7677-7687.

[18] M. Aldhoun, A. Massi, A. Dondoni, J. Org. Chem. 2008, 73, 9565-9575.

[19] C. Aydillo, C. D. Navo, J. H. Busto, F. Corzana, M. M. Zurbano, A. Avenoza, J. M. Peregrina, J. Org. Chem. 2013, 78, 10968-10977.

[20] B. Richichi, B. Thomas, M. Fiore, A. Bosco, H. Qureshi, C. Nativi, O. Renaudet, L. BenMohamed, Angew. Chem. Int. Ed. 2014, 53, 11917-11920: Angew. Chem, 2014, 126, 12111 .

[21] For recent reviews; a) T. B. Nguyen, A. Martel, C. Gaulon, R. Dhal, G. Dujardin, Org. Prep. Proced. Int. 2010, 42,387-431; b) T. B. Nguyen, A. Martel, C. Gaulon-Nourry, R. Dhal, G. Dujardin, Org. Prep. Proced int. 2012,44, 1-81; c) M. Berthet, T. Cheviet, G. Dujardin, I. Parrot, J. Martinez, Chem. Rev. 2016, 116, 15235-15283; d) A. Brandi, F. Cardona, S. Cicchi, F. Cordero, A. Goti in [3+2] Dipolar Cycloadditions of Cyclic Nitrones with Alkenes, Org. Reaci. 2017, p. 94.

[22] With exo-glycals, see for example: a) X. Li, H. Takahashi, H. Ohtake, 5. Ikegami, Heterocycles 2003, 59, 547-551; b) X. Li, H. Takahashi, H. Ohtake, 5. Ikegami, Tetrahedron Lett. 2004, 45, 4123-4126; c) H. Oukani, N. Pellegrini-Moise, O. Jackowski, F. Chrétien, Y. Chapleur, Carbohydr. Res. 2013. $381,205-214$

[23] With C.allylglycoside, see for example: B. Westernann, A. Walter, U. Flörke, H.-J. Altenbach, Org. Lett. 2001, 3, 1375-1378.

[24] With 5.C.vinylgalactoside, see for example: a) F. Duff, V. Vivien, R. Wightman, Chem. Commun 2000, 2127-2128: b) N. G. Argyropoulos, V. C. Sarli, Tetrahedron Lett. 2004, 45, 4237-44240

[25] 1,3-dipolar cycloaddition with C-vinylglycosides was menroned in pef (23: but no yield and no analytical data were given.

[26] F. Rouzier, R. Sillé, A. Nourry, A. Tessier, M. Pipelier, S. Guillarme, Synthesis 2019, 51, 2484-2488

[27] T. B. Nguyen, A. Martel, R. Dhal, G. Dujardin, Synthesis 2009, 3174-3176.

[28] K. Ranieri, M. Conradi, P.Y. Chavant, V. Blandin, C. Barner-Kowollik, T. Junkers, Aust. J. Chem. 2012, 65, 1110-1116.

[29] a) N. Katagiri, M. Okada, Y. Morishita, C. Kaneko, Chem. Commun. 1996 2137-2138; b) N. Katagiri, M. Okada. C. Kaneko, T. Furuya, Tetrohedron Lett. 1996, 11, 1801-1804.

[30] K. Aouadi, M. Msaddek, J.P. Praly, Tetrahedron 2012, 68, 1762-1768.

[31] Deprotection of benzyl groups of cycloadduct $7 \mathrm{~b}$ was performed using cyclohexene, $\mathrm{Pd}(\mathrm{OH})_{2} / \mathrm{C}$ in ethanol under reflux for $6 \mathrm{~h}$ leading to unpro. tected cycloadduct in $80 \%$ yield.

[32] M. J. Frisch, G.W. Trucks, H. B. Schlegel, G. E. Scuseria, M. A. Robb, J. R. Cheeseman, G. Scalmani, V. Barone, B. Mennucci, G. A. Petersson, H. Na. katsuji, M. Caricato, X. Li, H. P. Hratchian, A. F. Izmaylov, J. Bloino, G. Zheng, J. L. Sonnenberg, M. Hada, M. Ehara, K. Toyota, R. Fukuda, J. Hasegawa, M. Ishida, T. Nakajima, Y. Honda, O. Kitao, H. Nakai, T. Vreven, J. A. Montgomery Jr., J. E. Peralta, F. Ogliaro, M. Bearpark, J. J. Heyd, E. Broth. 
ers, K. N. Kudin, V. N. Staroverov, R. Kobayashi, J. Normand, K. Raghavachari, A. Rendell, J. C. Burant, S. S. lyengar, J. Tomasi, M. Cossi, N. Rega, J. M. Millam, M. Klene, J. E. Knox, J. B. Cross, V. Bakken, C. Adamo, J. Jaramillo, R. Gomperts, R. E. Stratmann, O. Yazyev, A. J. Austin, R. Cammi, C. Pomelli, J. W. Ochterski, R. L. Martin, K. Morokuma, V. G. Zakrzewski, G. A. Voth, P. Salvador, J. J. Dannenberg, S. Dapprich, A. D. Daniels, O. Farkas, J. B. Foresman, J. V. Ortiz, J. Cioslowski, D. J. Fox, Gaussian 09. Revision D.01, Gaussian, Inc., Wallingford CT, 2013.
[33] Y. Zhao, 0. G. Truhlar, Theor. Chem. Acc. 2008, 120, 215-241.

34] A. V. Marenich, C. J. Cramer, D. G. Truhlar, 1. Phys. Chem. A J. Phys. Chem B J. Phys. Chem A 2009, 113,6378-6396

[35] For more details about DFT calculation data, see Supporting Information

Received: August 25, 2020 